\title{
Light Majorana neutrinos in (semi)invisible meson decays
}

\author{
Blaž Bortolato $^{1, \mathrm{a}}$, Jernej F. Kamenik ${ }^{1,2, \mathrm{~b}}$ (D) \\ ${ }^{1}$ Jožef Stefan Institute, Jamova 39, 1000 Ljubljana, Slovenia \\ ${ }^{2}$ Faculty of Mathematics and Physics, University of Ljubljana, Jadranska 19, 1000 Ljubljana, Slovenia
}

Received: 10 August 2020 / Accepted: 19 April 2021 / Published online: 4 May 2021

(C) The Author(s) 2021

\begin{abstract}
We reconsider decays of pseudoscalar mesons $(P)$ to neutrino pairs and possibly additional photons in presence of (light) Majorana neutrinos. For this purpose we derive a model-independent general parametrization of neutrino mass matrices with physically interpretable and irreducible set of parameters. The parametrization is valid for any number of neutrinos and interpolates smoothly between the heavy Majorana and the (pseudo)Dirac neutrino limits. We apply the new parametrization to the study of $P \rightarrow v v$ and $P \rightarrow \nu v \gamma$ decays within the SM extended by additional singlet fermions. We update the SM predictions for the branching ratios of $B_{s, d} \rightarrow \nu \nu \gamma$ and discuss the sensitivity of the $B_{s, d} \rightarrow E_{\text {miss }}(\gamma)$ decays to neutrino mass and mixing parameters.
\end{abstract}

\section{Introduction}

The discovery of neutrino oscillations [1] implies the existence of at least two massive neutrino species. On the other hand, many theoretical models of neutrino mass generation, including the simplest canonical see-saw mechanism [26], predict the existence of additional electromagnetically neutral massive fermions. In general, the neutrino spectrum consists of $3+n_{N}$ fermions. Three of them are the so-far observed standard model (SM) like $\left(v_{j}^{M}\right)$ neutrinos. Possible additional $n_{N}$ massive neutrinos $\left(N^{M}\right)$ have not yet been observed. In the following and without loss of generality we assume them to be of Majorana type. ${ }^{1}$ In the last few decades many different mechanisms have been proposed to explain the smallness of the observed neutrino masses. In

\footnotetext{
a e-mail: blaz.bortolato@ijs.si

b e-mail: jernej.kamenik@cern.ch (corresponding author)

1 The model of Dirac SM-like neutrinos is a special case with $n_{N}=3$ and with all Majorana mass terms set to zero. Its spectrum consists of 3 Dirac neutrino fields, which can be written as a superposition of $v_{j}^{M}$ and $N_{j}^{M}$ fields.
}

the canonical see-saw mechanism for example, heavy $N_{k}^{M}$ neutrinos induce small Majorana masses for the observed $v_{j}^{M}$ neutrinos via their Yukawa interactions. In this scenario $N_{k}^{M}$ neutrinos are typically too heavy to be directly produced in terrestrial experiments [7]. On the other hand, in models with approximate lepton number conservation, these new degrees of freedom could also naturally appear at low energies, see e.g. [8]. In fact there are several circumstantial motivations for considering additional light $N_{k}^{M}$ neutrinos. Massive neutral fermions which are long lived enough compared to the age of the universe and have mass in the range $2 \mathrm{keV} \lesssim m_{N_{k}} \lesssim 5 \mathrm{keV}[9,10]$ are good warm dark matter candidates [11-14]. Additional $N_{k}^{M}$ neutrinos with masses in the range $1 \mathrm{GeV} \leq m_{N_{k}} \ll 100 \mathrm{GeV}$ are also predicted in models of cosmological Baryon asymmetry generation through neutrino oscillations $[15,16]$. Finally, persistent tensions in the interpretation of some neutrino oscillation experiments and cosmological observations might imply the existence of additional $N_{k}^{M}$ neutrinos with masses at the $\mathrm{eV}$ scale, see e.g. [17].

An important aspect of neutrino mass model building involves consistently taking into account existing experimental information on low energy neutrino masses and mixings. In principle these inputs can be used to reduce the number of free model parameters. In practice however, this requires a detailed a priori knowledge of how elements of neutrino mass and mixing matrices are connected with each other. In the limit of heavy $N_{k}^{M}$ neutrinos such connections are given explicitly by the Casas-Ibara parametrization [18]. In the last few years, more general parameterizations have been proposed, which do not rely on expansions in small mass ratios and are thus valid away from the limit of heavy $N_{k}^{M}$ neutrinos. To date such parametrizations have been found for the case of two [19,20] or three [21-23] additional $N_{k}^{M}$ neutrinos. Most recently, a master parametrization applicable for the most general case including neutrino mass generation beyond see-saw models has also been proposed [24]. However, the generality comes with several drawbacks: its param- 
eters lack intuitive physical interpretability, the connections between the $N_{k}^{M}$ neutrino masses and the SM-like neutrino Yukawa couplings are somewhat obscured. In this paper we build upon and extend previous work [19,23] and derive a model-independent general parametrization of the neutrino mass matrices that covers and interpolates between all seesaw like scenarios, including the heavy Majorana mass limit and the pseudo-Dirac case for any number of additional $N_{k}^{M}$ neutrinos (see e.g. Ref. [25] for an explicit model realization of such a scenario). Its main purpose is to better map out the possible neutrino mass parameter space and to help create a consistent picture of $N_{k}^{M}$ neutrinos at low energies, which is a starting point for developing UV neutrino mass models. ${ }^{2}$

We demonstrate the usefulness of the parametrization using the example of $P \rightarrow v v$ and $P \rightarrow \nu v \gamma$ decays, previously studied in Ref. [27] in the context of light dark matter searches, where $P$ is a neutral pseudoscalar meson and $v$ includes both $v_{j}^{M}$ as well as possibly $N_{k}^{M}$ if kinematically allowed. We estimate the contribution of these two decay topologies to the effective invisible decay widths of neutral mesons (assuming $N_{k}^{M}$ are long lived enough to escape the detectors) and show how they are sensitive to neutrino mass and mixing parameters. Along the way we also update the theoretical predictions for $B_{s, d} \rightarrow v v \gamma$ in the SM using state-of-the-art inputs [28] for the relevant hadronic parameters and the associated uncertainties. The $P \rightarrow v v$ decays are helicity suppressed and therefore negligible in the SM with Dirac neutrinos [27] as well as in the limit of heavy $N_{k}^{M}$. However in models with light $N_{k}^{M}$, such that they can appear in the final state, their branching fractions can become significant. On the other hand $P \rightarrow v v v v$ decays are not helicity suppressed [29], however their contributions to the invisible $P$ decay widths turn out (within our assumptions) to be completely negligible. Experimentally, the best sensitivity is expected from $B_{s, d}$ meson decays into unobserved decay products (registered as missing energy $E_{\text {miss }}$ in the detector) which have already been searched for by the Belle [30] and $\mathrm{BaBar}$ [31] collaborations. At present the tightest upper limit of $\mathcal{B r}\left(B_{d} \rightarrow E_{\text {miss }}\right)<2.4 \times 10^{-5}$ at $90 \%$ confidence level is provided by BaBar [31]. While searches for invisible $B_{s}$ decays have not yet been attempted, they are planned at the Belle II experiment, which is also expected to improve significantly the upper bound on $\mathcal{B r}\left(B_{d} \rightarrow E_{\text {miss }}\right)$ [32].

The paper is organized as follows. In Sect. 2 we derive a general parametrization of neutrino mass matrices for an arbitrary number of additional massive fermionic singlets and explore the heavy Majorana neutrino limit and the pseudoDirac limit. We also present the basic properties of the parametrization and how these can be used to extract neu-

${ }^{2}$ We note that in UV models where $B-L$ is gauged (like in $U(1)_{B-L}[26]$ or left-right symmetric models $\left.[3,4]\right)$, anomaly cancellation requires exactly three right-handed neutrinos $\left(n_{N}=3\right)$. trino parameters from experiments. In Sect. 3 we study the $P \rightarrow v v$ and $P \rightarrow v v \gamma$ decays separately, estimate their contributions to the invisible decay widths of $B_{d, s}$ mesons, and discuss their dependence on the neutrino parameters. We summarize our findings in Sect. 4. Analytical expressions for the $P \rightarrow v v$ and $P \rightarrow v v \gamma$ decays as well as the details on the perturbative and non-perturbative QCD inputs used in this work are given in Appendix A, while Appendix B contains the details on the derivation of a lower bound on the Frobenius norm of the neutrino mixing matrix.

\section{Parametrizing neutrino masses and mixing in presence of light Majorana neutrinos}

\subsection{Setup and notation}

We consider a family of neutrino models at low energies which are described by the Lagrangian ${ }^{3} \mathcal{L}=\mathcal{L}^{\mathrm{SM}}+\mathcal{L}^{N}$, where $\mathcal{L}^{\mathrm{SM}}$ is the Standard Model (SM) Lagrangian and $\mathcal{L}^{N}$ is given by:

$$
\begin{aligned}
\mathcal{L}^{\mathrm{N}}= & -\sum_{a=1}^{3} \sum_{b=1}^{n} \overline{v_{a L}}\left(M_{D}\right)_{a b} N_{b R}+\text { h.c. } \\
& -\frac{1}{2} \sum_{b=1}^{n} \sum_{b^{\prime}=1}^{n} \overline{\left(N_{b R}\right)^{c}}\left(M_{M}\right)_{b b^{\prime}} N_{b^{\prime} R}+\text { h.c. } \\
& +\sum_{b=1}^{n} \overline{N_{b R}} \mathrm{i} \gamma^{\mu} \partial_{\mu} N_{b R} .
\end{aligned}
$$

The first term is the Dirac mass term where the Yukawa coupling matrix $y$ is implicit in $M_{D}=v / \sqrt{2} y$, where $v$ is the Higgs VEV. The second term is the Majorana mass term of chiral right-handed neutrinos $N_{b R}$. The mass matrices of models which preserve SM local symmetries and are renormalizable (chiral left handed neutrino mass terms are forbidden) form a symmetric block matrix $M$ of the form ${ }^{4}$

$$
\begin{aligned}
\mathcal{L} \supset & -\frac{1}{2}\left(\overline{\nu_{L}} \overline{\left(N_{R}\right)^{c}}\right)\left(\begin{array}{cc}
0_{3 \times 3} & \left(M_{D}\right)_{3 \times n} \\
\left(M_{D}^{T}\right)_{n \times 3} & \left(M_{M}\right)_{n \times n}
\end{array}\right)\left(\begin{array}{c}
\left(v_{L}\right)^{c} \\
N_{R}
\end{array}\right) \\
& + \text { h.c.. }
\end{aligned}
$$

The matrix $M$ can be diagonalized by the unitary matrix $L$ in the following way

$$
M_{\text {diag }}=L^{\dagger}\left(\begin{array}{cc}
0 & M_{D} \\
M_{D}^{T} & M_{M}
\end{array}\right) L^{*}=\left(\begin{array}{cc}
D_{v} & 0 \\
0 & D_{N}
\end{array}\right), \text { where }
$$

\footnotetext{
${ }^{3}$ We use the formalism presented in Ref. [33]. A comprehensive discussion of Dirac, Weyl and Majorana fields is given in Ref. [34].

${ }^{4}$ The symmetric nature of $M$ is typical for see-saw like models of neutrino mass generation and is central to our parametrization. For more general scenarios leading to non-symmetric $M$, the parametrization of Ref. [24] applies.
} 


$$
L=\left(\begin{array}{cc}
U_{3 \times 3} & V_{3 \times n} \\
X_{n \times 3} & Y_{n \times n}
\end{array}\right)
$$

Here $D_{v}=\operatorname{diag}\left(m_{v_{1}}, m_{v_{2}}, m_{v_{3}}\right)$ is the diagonal mass matrix of SM-like neutrinos $\left(v_{j}^{M}=\left[v_{j L}+\left(v_{j L}\right)^{c}\right]_{m}\right)$ and $D_{N}=$ $\operatorname{diag}\left(m_{N_{1}}, \ldots, m_{N_{n}}\right)$ is the diagonal mass matrix of $N$ neutrinos $\left(N_{k}^{M}=\left[N_{k R}+\left(N_{k R}\right)^{c}\right]_{m}\right)$, where $\left(v_{j L}\right)_{m}$ and $\left(N_{k R}\right)_{m}$ are the mass eigenstates which are connected to the gauge interaction eigenstates via

$\left(\begin{array}{c}v_{L} \\ \left(N_{R}\right)^{c}\end{array}\right)=L\left(\begin{array}{c}v_{L} \\ \left(N_{R}\right)^{c}\end{array}\right)_{\mathrm{m}}$.

Without loss of generality, all diagonal elements of $D_{v}$ and $D_{N}$ can be taken as real (via suitable unphysical phase rotations of the neutrino fields). With this notation one can write down the interaction terms in the Lagrangian expressed by $v_{j}^{M}$ and $N_{k}^{M}$ neutrino mass eigenstates and by $l_{m} \in\{e, \mu, \tau\}$ charged lepton mass eigenstates,

$$
\begin{aligned}
\mathcal{L} \supset & -\frac{g W_{\mu}^{+}}{\sqrt{2}} \sum_{l_{m} \in\{e, \mu, \tau\}}\left(\sum_{j=1}^{3}\left(U^{\dagger} O_{L}\right)_{j m} \overline{v_{j}^{M}} \gamma^{\mu} P_{L} l_{m}\right. \\
& \left.+\sum_{k=1}^{n}\left(V^{\dagger} O_{L}\right)_{k m} \overline{N_{k}^{M}} \gamma^{\nu} P_{L} l_{m}\right)+ \text { h.c. } \\
& -\frac{g Z_{\mu}}{2 \cos \theta_{W}}\left(\sum_{j, j^{\prime}=1}^{3}\left(U^{\dagger} U\right)_{j j^{\prime}} \overline{v_{j}^{M}} \gamma^{\mu} P_{L} v_{j^{\prime}}^{M}\right. \\
& \left.+\sum_{k, k^{\prime}=1}^{n}\left(V^{\dagger} V\right)_{k k^{\prime}} \overline{N_{k}^{M}} \gamma^{v} P_{L} N_{k^{\prime}}^{M}\right) \\
& -\frac{g Z_{\mu}}{2 \cos \theta_{W}}\left(\sum_{j=1}^{3} \sum_{k=1}^{n}\left(U^{\dagger} V\right)_{j k} \overline{v_{j}^{M}} \gamma^{\mu} P_{L} N_{k}^{M}+\text { h.c. }\right)
\end{aligned}
$$

where $O_{L}$ and $O_{R}$ are unitary matrices diagonalizing the mass matrix $M^{l}$ of the charged leptons via the biunitary transformation $O_{L}^{\dagger} M^{l} O_{R}=\operatorname{diag}\left(m_{e}, m_{\mu}, m_{\tau}\right)$ and $P_{L, R}=$ $\left(1 \mp \gamma_{5}\right) / 2$.

\subsection{Derivation}

In this section we consider a model with $n_{v}$ neutrinos $v_{j}^{M}$ and $n_{N}$ neutrinos $N_{k}^{M}$. Matrices $U, V, X$ and $Y$ clearly depend on the neutrino masses, thus it is appropriate to have a simple parametrization of these matrices in terms of physical neutrino parameters. Parametrization of this type can be derived with a few simple steps. We start by decomposing the $V$ matrix into

$V=\left\{\begin{array}{l}g S: n_{N}>n_{v}, \\ g P: n_{N} \leq n_{v}\end{array}\right.$

where $g$ is a $n_{v} \times n_{v}$ complex matrix, $S$ is given by $S_{n_{v} \times n_{N}}=$ $\left[I_{n_{v} \times n_{v}}, \hat{S}_{n_{v} \times\left(n_{N}-n_{v}\right)}\right]$ and $P_{n_{v} \times n_{N}}$ is a projection matrix which in the case of a normal hierarchy of $v_{j}^{M}$ neutrino masses is given by

$P=\left(\begin{array}{c}0_{\left(n_{v}-n_{N}\right) \times n_{N}} \\ I_{n_{N} \times n_{N}}\end{array}\right)$.

The $\hat{S}$ matrix is a general complex $n_{v} \times\left(n_{N}-n_{v}\right)$ matrix. Its elements can be chosen freely. By using the definition of the transition matrix, $L M_{\text {diag }} L^{T}=M$ one obtains the relation

$U D_{\nu} U^{T}+V D_{N} V^{T}=0$

which, depending on $n_{N}$, can be rewritten as

$$
\begin{aligned}
& U D_{v}^{1 / 2} D_{v}^{1 / 2} U^{T} \\
& =g\left(-S D_{N} S^{T}\right)^{1 / 2}\left(-S D_{N} S^{T}\right)^{1 / 2} g^{T}: n_{N}>n_{\nu}, \\
& U D_{\nu} U^{T} \\
& =g P\left(-D_{N}\right)^{1 / 2}\left(-D_{N}\right)^{1 / 2} P^{T} g^{T} \quad: n_{N} \leq n_{v} \text {. }
\end{aligned}
$$

Equation (9a) is already in the desired form. With a few assumptions it can be written as $R R^{T}=I$, where $R$ is an invertible orthogonal complex squared matrix, which connects $U D_{v}^{1 / 2}$ with $g\left(-S D_{N} S^{T}\right)^{1 / 2}$. On the other hand Eq. (9b) is not yet of the desired form. In the special case $n_{N}=n_{v}$ the projection matrix $P$ becomes the identity matrix, and we can decompose the left-hand side of Eq. (9b) into $U D_{v} U^{T}=U D_{\nu}^{1 / 2} D_{v}^{1 / 2} U^{T}$. Notice that on both sides all matrices have the same shape. In the case $n_{N}<n_{v}$ the diagonal mass matrix $D_{v}$ is not invertible. This can be seen directly by looking at the block mass matrix $M$,

$M=\left(\begin{array}{cc}0_{n_{v} \times n_{v}} & \left(M_{D}\right)_{n_{v} \times n_{N}} \\ \left(M_{D}^{T}\right)_{n_{N} \times n_{v}} & \left(M_{M}\right)_{n_{N} \times n_{N}}\end{array}\right)$.

Of the first $n_{v}$ rows, maximally $n_{N}$ are independent. Similarly, of the last $n_{N}$ rows, also maximally $n_{N}$ are independent. Therefore one can construct at least $n_{v}+n_{N}-2 n_{N}=n_{v}-n_{N}$ independent eigenvectors for the matrix $M$ with zero eigenvalues. From here, there are maximally $n_{N}$ non zero masses $m_{v_{j}}$ in $D_{v}$. This property can be written as

$D_{v}=P\left(P^{T} D_{v} P\right) P^{T}$

where $P^{T} D_{\nu} P$ is a $n_{N} \times n_{N}$ diagonal matrix of all non zero eigenvalues of $D_{\nu}$, therefore an invertible positive definite 
matrix. By specifying $D_{v}$ one can use this property to find $P$. Using Eq. (11) one can finally rewrite the left-hand side of Eq. (9b) in terms of $n_{N} \times n_{N}$ matrices,

$$
\begin{aligned}
& \left(\tilde{P}^{T} U P\right)\left(P^{T} D_{\nu}^{1 / 2} P\right)\left(P^{T} D_{\nu}^{1 / 2} P\right)\left(P^{T} U \tilde{P}\right) \\
& \quad=\left(\tilde{P}^{T} g P\right)\left(-D_{N}\right)^{1 / 2}\left(-D_{N}\right)^{1 / 2}\left(P^{T} g^{T} \tilde{P}\right) .
\end{aligned}
$$

Here we have multiplied the equation by a $n_{v} \times n_{N}$ arbitrary matrix $\tilde{P}$ from the right-hand side and by $\tilde{P}^{T}$ from the lefthand side.

At this point we assume that all matrices, which are products of matrices inside brackets in Eqs. (12) and (9a), are invertible, $D_{N}$ matrix is invertible for all pairs $\left(n_{v}, n_{N}\right)$ and that matrices $U$ and $g$ are also invertible in the case $n_{v}<n_{N}$. Cases in which these assumptions do not hold can still be handled by the parametrization we are deriving, by taking appropriate limits. Within our assumptions, both Eqs. (9a) and (12) can be expressed in the form $R^{T} R=I$ or $R R^{T}=I$ equivalently, where $R$ is a $\min \left(n_{v} \times n_{v}, n_{N} \times n_{N}\right)$ general complex orthogonal matrix. It links $U$ and $V$ matrices through

$V=U Q$,

where the $n_{v} \times n_{N}$ matrix $Q$ is given by

$Q= \begin{cases}D_{\nu}^{1 / 2} R\left(-S D_{N} S^{T}\right)^{-1 / 2} S: n_{N}>n_{\nu}, \\ D_{v}^{1 / 2} P R\left(-D_{N}\right)^{-1 / 2} & : n_{N} \leq n_{\nu} .\end{cases}$

In case $n_{v}>n_{N}$ one obtains the relation $\tilde{P}^{T}(V-U Q)=0$ which leads to $V=U Q$, since $\tilde{P}^{T}$ is arbitrary up to the above assumptions. Eq. (13) together with the unitarity condition $L L^{\dagger}=I$ finally leads to the desired parametrization of $U$ and $V$ matrices

$U U^{\dagger}+V V^{\dagger}=I$,

$U \sqrt{I+Q Q^{\dagger}} \sqrt{I+Q Q^{\dagger}} U^{\dagger}=I$,

$U=A\left(I+Q Q^{\dagger}\right)^{-1 / 2}$.

Here $A$ is a $n_{v} \times n_{v}$ unitary matrix. The $I+Q Q^{\dagger}$ hermitian matrix is positive definite, which means that it has precisely one positive definite square root which is also invertible. Parametrizations of $X$ and $Y$ matrices are similarly obtained from the unitary condition $L L^{\dagger}=I$ using the above derived results.

\subsection{Main formulae}

Below we give the full set of equations which define the parametrization of the neutrino mixing matrices:

$U=A\left(\sqrt{I+Q Q^{\dagger}}\right)^{-1}$,

$$
\begin{aligned}
& V=A\left(\sqrt{I+Q Q^{\dagger}}\right)^{-1} Q, \\
& X=-B\left(\sqrt{I+Q^{\dagger} Q}\right)^{-1} Q^{\dagger}, \\
& Y=B\left(\sqrt{I+Q^{\dagger} Q}\right)^{-1},
\end{aligned}
$$

where $A_{n_{v} \times n_{v}}$ and $B_{n_{N} \times n_{N}}$ are unitary matrices and Q is defined by

$Q= \begin{cases}-\mathrm{i} D_{v}^{1 / 2} R\left(S D_{N} S^{T}\right)^{-1 / 2} S: n_{N}>n_{\nu}, \\ -\mathrm{i} D_{v}^{1 / 2} P R D_{N}^{-1 / 2} & : n_{N} \leq n_{\nu} .\end{cases}$

The $M_{D}=v / \sqrt{2} y$ and $M_{M}$ matrices are therefore parametrized via

$$
\begin{aligned}
& M_{D}=U D_{\nu} X^{T}+V D_{N} Y^{T}, \\
& M_{M}=X D_{\nu} X^{T}+Y D_{N} Y^{T},
\end{aligned}
$$

where $D_{v}$ and $D_{N}$ are diagonal neutrino mass matrices $D_{v}=$ $\operatorname{diag}\left(m_{v_{1}}, \ldots, m_{v_{n_{v}}}\right)$ and $D_{N}=\operatorname{diag}\left(m_{N_{1}}, \ldots, m_{N_{N_{n}}}\right)$ defined up to arbitrary phases (for each diagonal element). The derived parametrization expresses neutrino mixing matrices appearing in the Lagrangian in terms of unitary matrices $A$ and $B$, complex orthogonal matrix $R$, neutrino masses contained in $D_{v}$ and $D_{N}$ and in case $n_{N}>n_{v}$ also a general complex matrix $\hat{S}$ which is hidden inside $S=\left[I_{n_{\nu} \times n_{v}}, \hat{S}_{n_{\nu} \times\left(n_{N}-n_{v}\right)}\right]$. The projector $P$ in case $n_{v} \geq n_{N}$ must be chosen such that $P^{T} D_{\nu} P$ is a diagonal matrix of all non zero eigenvalues of $D_{v}$ and that the relation $P P^{T} D_{v} P P^{T}=D_{v}$ holds. If $n_{v}>n_{N}$, the choice of $P$ depends on the hierarchy of $v_{j}^{M}$ neutrino masses.

\subsection{Physical case $n_{v}=3$}

In the following we discuss the explicit form of our parametrization, it's limits and parameter counting, for the realistic case $n_{v}=3$ and various possible choices of $n_{N}$.

In the case $n_{v}=n_{N}=3$ previously studied in Ref. [23] the $R$ matrix can be parametrized with 3 complex angles:

$R=\left(\begin{array}{ccc}c_{1} & \pm s_{1} & 0 \\ -s_{1} & \pm c_{1} & 0 \\ 0 & 0 & 1\end{array}\right)\left(\begin{array}{ccc}c_{2} & 0 & \pm s_{2} \\ 0 & 1 & 0 \\ -s_{2} & 0 & \pm c_{2}\end{array}\right)\left(\begin{array}{ccc}1 & 0 & 0 \\ 0 & c_{3} & \pm s_{3} \\ 0 & -s_{3} & \pm c_{3}\end{array}\right)$

where $c_{j}=\cos \left(\phi_{j}+\mathrm{i} \theta_{j}\right)$ and $s_{j}=\sin \left(\phi_{j}+\mathrm{i} \theta_{j}\right)$ with $\phi_{j} \in[0,2 \pi)$ and $\theta_{j} \in \mathbb{R}$ for $j \in\{1,2,3\}$. Free signs in each of the three matrices must be equal (both + or both in each matrix). The projector $P$ in this case reduces to the identity matrix.

In the case $n_{v}=3$ with $n_{N}=2$ previously studied in Ref. [19] one of the $v_{j}^{M}$ neutrinos is massless, thus the $D_{v}$ 
matrix can be parametrized by $D_{v}=\operatorname{diag}\left(0, m_{v_{2}}, m_{v_{3}}\right)$ in case of normal hierarchy $(\mathrm{NH})$ or by $D_{v}=\operatorname{diag}\left(m_{v_{1}}, 0, m_{v_{3}}\right)$ in case of inverted hierarchy (IH). In both scenarios the $R$ matrix is described by one complex angle:

$R=\left(\begin{array}{rr}\cos (\phi+\mathrm{i} \theta) & \pm \sin (\phi+\mathrm{i} \theta) \\ -\sin (\phi+\mathrm{i} \theta) & \pm \cos (\phi+\mathrm{i} \theta)\end{array}\right)$,

where $\phi \in[0,2 \pi)$ and $\theta \in \mathbb{R}$. As before the free signs in the $R$ matrix must be equal (both + or both - ). The projector $P$ for $\mathrm{NH}(\mathrm{IH})$ is given by:

$$
P^{\mathrm{NH}}=\left(\begin{array}{ll}
0 & 0 \\
1 & 0 \\
0 & 1
\end{array}\right) \quad \text { and } \quad P^{\mathrm{IH}}=\left(\begin{array}{ll}
1 & 0 \\
0 & 1 \\
0 & 0
\end{array}\right)
$$

Also, in the case $n_{v}>n_{N}$ one can rename the $P R$ matrix to $R_{n_{v} \times n_{N}}$, since the projector $P$ is always multiplied by $R$ from the right-hand side.

On the other hand, matrix $B$ is a general $n_{N} \times n_{N}$ unitary matrix for all pairs $\left(n_{v}, n_{N}\right)$, while matrix $A$ is a general $n_{v} \times n_{v}$ unitary matrix only if $n_{v} \leq n_{N}$, since if $n_{v}>n_{N}$ not all generators of $\mathrm{U}\left(n_{v}\right)$ are required to form $A$ so that the $\left(M_{D}\right)_{n_{v} \times n_{N}}$ and $\left(M_{M}\right)_{n_{N} \times n_{N}}$ matrices are fully parametrized with all $n_{N}^{2}+n_{N}\left(2 n_{v}+1\right)$ parameters. The number of real parameters for each matrix which appears in the derived parametrization for the case $n_{v}=3$ is given in Table 1 .

\subsection{Heavy $N_{k}$ limit}

In the limit where $\left\|D_{v}\right\| /\left\|D_{N}\right\| \ll 1$ and $\|Q\| \ll 1$ the parametrization simplifies as $U \approx A, V \approx A Q, X \approx$ $-B Q^{\dagger}, Y=B$ resulting in

$M_{D}= \begin{cases}-\mathrm{i} A D_{\nu}^{1 / 2} R\left(S D_{N} S^{T}\right)^{-1 / 2} S D_{N} B^{T}: n_{N}>n_{\nu} \\ \mathrm{i} A D_{\nu}^{1 / 2} P R D_{N}^{1 / 2} B^{T} & : n_{N} \leq n_{\nu}\end{cases}$

and

$M_{M} \approx B D_{N} B^{T}$

In case $n_{N} \leq n_{v}$ we immediately recognize the original Casas-Ibara parametrization [18]. By combining the expressions of $M_{D}$ and $M_{M}$ matrices one can also construct the well known effective $v_{j}^{M}$ neutrino mass matrix

$M_{v}^{\text {effective }} \equiv-M_{D} M_{M}^{-1} M_{D}^{T} \approx A D_{v} A^{T}$.

This formula is valid for all pairs $\left(n_{v}, n_{N}\right)$. In this scenario the PMNS matrix which is given by $U_{\mathrm{PMNS}}=O_{L}^{\dagger} U \approx O_{L}^{\dagger} A$ (see Eq. (5)) is approximately unitary. To clarify, the PMNS matrix maps fields of observed neutrinos from the mass basis into the flavor (gauge) basis. In the heavy neutrino limit low energy processes can only involve $v_{j}^{M}$ neutrinos.

\subsection{Dirac neutrino limit}

In the scenario with $n \equiv n_{v}=n_{N}$ and $M_{M}=0$ neutrinos can be described by Dirac fields (linear combinations of Majorana fields which are not Majorana fields). The condition $M_{M}=0$ is equivalent to $R^{\dagger}\left|D_{\nu}\right|^{2} R^{*}=\left|D_{N}\right|^{2}$. The phases in front of neutrino masses are arbitrary and do not affect any measurable quantities. Therefore we can fix the phases by choosing $D \equiv D_{v}=D_{N}$, where $D$ is a positive definite mass matrix. From here, one finds $Q=-\mathrm{i} R$, where $R=\operatorname{diag}( \pm 1, \ldots, \pm 1)$. Signs are arbitrary and independent of each other. The transition matrix $L$ then takes the following form

$L=\frac{1}{\sqrt{2}}\left(\begin{array}{cc}A & -\mathrm{i} A R \\ -\mathrm{i} B R & B\end{array}\right)$

At this point one can diagonalize $M_{D}$ through a biunitary transformation

$M_{D}=-\mathrm{i} A R D B^{T}$

Therefore the mass term in the Lagrangian can be written as

$\mathcal{L} \supset-\bar{v}^{D} D v^{D}$

where

$v^{D}=\frac{1}{\sqrt{2}}\left(i R v_{j}^{M}+N_{j}^{M}\right)$

are Dirac neutrino fields (not Majorana fields). One can use this equation to define $v_{j}^{M}$ and $N_{j}^{M}$ Majorana fields starting from the Dirac field. Such definition of Majorana fields can then be applied outside the Dirac limit. In this way, the obtained model is fully consistent with both the general Majorana neutrino model (outside the Dirac limit) as well as with the Dirac neutrino model (in the Dirac limit). We use this model in Sect. 3. Finally, the Lagrangian (for the case $n=3$ ) can be written as

$$
\begin{aligned}
\mathcal{L} \supset \sum_{k=1}^{3} \overline{v_{k}^{D}}\left(\mathrm{i} \gamma^{\mu} \partial_{\mu}-D\right) v_{k}^{D} \\
+\frac{g}{2 \cos \left(\theta_{W}\right)} Z_{\mu} \sum_{k=1}^{3} \overline{v_{k}^{D}} \gamma^{\mu} P_{L} v_{k}^{D} \\
+\frac{g}{\sqrt{2}} W_{\mu}^{+} \sum_{l_{m} \in\{e, \mu, \tau\}} \sum_{k=1}^{3}\left(A^{\dagger} O_{L}\right)_{k m} \overline{v_{k}^{D}} \gamma^{\mu} P_{L} l_{m}+\text { h.c.. }
\end{aligned}
$$


Table 1 Number of parameters in the derived parametrization of the neutrino mass matrices of the extended SM with $n_{v}=3$ and $n_{N}=n$ additional chiral right-handed neutrinos. Note that interactions between

\begin{tabular}{llllllll}
\hline$n$ & $\left(D_{\nu}\right)_{3 \times 3}$ & $\left(D_{N}\right)_{n \times n}$ & $R_{\min (n \times n, 3 \times 3)}$ & $\hat{S}_{3 \times(n-3)}$ & $A_{3 \times 3}$ & $B_{n \times n}$ & total $n^{2}+7 n$ \\
\hline$n=1$ & 1 & 1 & 0 & $/$ & 5 & 1 & 8 \\
$n=2$ & 2 & 2 & 2 & $/$ & 8 & 4 & 18 \\
$n=3$ & 3 & 3 & 6 & $/$ & 9 & 9 & 30 \\
$n>3$ & 3 & $n$ & 6 & $6(n-3)$ & 9 & $n^{2}$ & $n^{2}+7 n$ \\
\hline
\end{tabular}

neutrinos (both $v_{j}$ and $N_{k}$ ) and other SM particles do not depend on the $B_{n \times n}$ matrix
From the last equation one can recognize the (unitary) PMNS matrix as $U_{\mathrm{PMNS}}=O_{L}^{\dagger} A=\sqrt{2} O_{L}^{\dagger} U$. Its matrix elements are precisely the same as in the heavy neutrino limit if we keep $A$ matrix unchanged. However, now the observed neutrinos are 3 Dirac fermions which are specific linear combinations of $v_{j}^{M}$ and $N_{j}^{M}$ fields. Note that the measured values of the PMNS matrix can be directly related to the underlying neutrino parameters only in the heavy neutrino and (pseudo-)Dirac limits. Outside these two limits the precise relations are non-trivial, since the PMNS matrix is no longer unitary. However, results from experiments which measure the PMNS matrix elements can still be used to constrain parameters of the general low energy neutrino models.

\subsection{Scaling relations}

Matrices $U^{\dagger} U, U^{\dagger} V$ and $V^{\dagger} V$ satisfy the relation:

$\left\|U^{\dagger} U\right\|^{2}+2\left\|U^{\dagger} V\right\|^{2}+\left\|V^{\dagger} V\right\|^{2}=n_{\nu}$,

where $\left\|U^{\dagger} V\right\|^{2}=\sum_{j j^{\prime}}\left|\left(U^{\dagger} V\right)_{j j^{\prime}}\right|^{2}$ is the Frobenius norm and $n_{v}$ is the number of $v_{j}^{M}$ neutrinos. The property can be derived by a few simple steps. First define matrix $\mathcal{U}$ as

$\mathcal{U}=\left(\begin{array}{ll}U^{\dagger} U & U^{\dagger} V \\ V^{\dagger} U & V^{\dagger} V\end{array}\right)$

Its relevant properties are $\mathcal{U}^{\dagger}=\mathcal{U}$ and $\mathcal{U}^{2}=\mathcal{U}$. The last one holds due to the unitarity condition $U U^{\dagger}+V V^{\dagger}=I_{n_{v} \times n_{v}}$. From here, one gets $\sum_{E=1}^{n_{\nu}+n_{N}} \mathcal{U}_{C E} \mathcal{U}_{E D}=\mathcal{U}_{C D}$. Therefore

$\sum_{C, D=1}^{n_{v}+n_{N}}\left|\mathcal{U}_{C D}\right|^{2}=\sum_{C, D=1}^{n_{v}+n_{N}} \mathcal{U}_{C D} \mathcal{U}_{D C}=\operatorname{Tr}\{\mathcal{U}\}=n_{v}$

where the identity $\operatorname{Tr}\left\{V^{\dagger} V\right\}=\operatorname{Tr}\left\{V V^{\dagger}\right\}$ was used. By the definition of the Frobenius norm, relations like $\left|\left(U^{\dagger} V\right)_{j k}\right|<$ $\left\|U^{\dagger} V\right\|$ hold. These can be used to constrain matrix elements especially in scenarios with nearly degenerated $N_{k}^{M}$ neutrino masses. In the heavy Majorana mass limit $m_{v} / m_{N} \ll 1$
Frobenius norms can be used as direct measures of nonunitarity in the $3 \times 3$ light neutrino sector, complementary to the PMNS matrix $\left(U^{\dagger} O_{L}\right)$, since in this limit exact $3 \times 3$ unitarity implies $\left\|U^{\dagger} U\right\|=n_{\nu}$, while $\left\|U^{\dagger} V\right\|=\left\|V^{\dagger} V\right\|=0$.

In general $\left\|U U^{\dagger}\right\|,\left\|U V^{\dagger}\right\|$ and $\left\|V V^{\dagger}\right\|$ norms can be expressed as a function of eigenvalues $\mu_{j}$ of the $Q Q^{\dagger}$ matrix:

$$
\begin{aligned}
& \left\|U^{\dagger} U\right\|^{2}=\sum_{j=1}^{n_{v}} \frac{1}{\left(1+\mu_{j}\right)^{2}}, \\
& \left\|U^{\dagger} V\right\|^{2}=\left\|V^{\dagger} U\right\|^{2}=\sum_{j=1}^{n_{v}} \frac{\mu_{j}}{\left(1+\mu_{j}\right)^{2}}, \\
& \left\|V^{\dagger} V\right\|^{2}=\sum_{j=1}^{n_{v}} \frac{\mu_{j}^{2}}{\left(1+\mu_{j}\right)^{2}}
\end{aligned}
$$

where the first identity can be related to the determinant of the PMNS matrix defined in the $m_{v} / m_{N} \ll 1$ limit since $\operatorname{det}\left(U^{\dagger} U\right)=\prod_{j=1}^{n_{\nu}}\left(1+\mu_{j}\right)^{-1}$. Number of $v_{j}^{M}$ neutrinos is also number of required quantities to completely describe norms.

In a special case in which $n_{v}=n_{N}=3$ and neutrino masses are degenerated, $D_{v}=m_{v} I$ and $D_{N}=m_{N} I$, equations above can be simplified,

$$
\begin{aligned}
\left\|U^{\dagger} U\right\|^{2}= & \frac{1}{\left(1+\frac{m_{v}}{m_{N}}\right)^{2}} \\
& +\frac{1}{\left(1+\frac{m_{v}}{m_{N}} \lambda\right)^{2}}+\frac{1}{\left(1+\frac{m_{v}}{m_{N}} \frac{1}{\lambda}\right)^{2}}, \\
\left\|U^{\dagger} V\right\|^{2}= & \frac{m_{v}}{m_{N}}\left(\frac{1}{\left(1+\frac{m_{v}}{m_{N}}\right)^{2}}\right) \\
& \left.+\frac{\lambda}{\left(1+\frac{m_{v}}{m_{N}} \lambda\right)^{2}}+\frac{\frac{1}{\lambda}}{\left(1+\frac{m_{v}}{m_{N}} \frac{1}{\lambda}\right)^{2}}\right), \\
\left\|V^{\dagger} V\right\|^{2}= & \left(\frac{m_{v}}{m_{N}}\right)^{2}\left(\frac{1}{\left(1+\frac{m_{v}}{m_{N}}\right)^{2}}\right.
\end{aligned}
$$




$$
\left.+\frac{\lambda^{2}}{\left(1+\frac{m_{v}}{m_{N}} \lambda\right)^{2}}+\frac{\frac{1}{\lambda^{2}}}{\left(1+\frac{m_{\nu}}{m_{N}} \frac{1}{\lambda}\right)^{2}}\right)
$$

where $\lambda$ is the largest eigenvalue of the $R^{\dagger} R$ matrix. Properties used to derive these equations are described in Appendix B. In this special case norms depend only on $\lambda \in[1, \infty)$ and $m_{v} / m_{N}$ ratio. However if $m_{v} / m_{N} \ll 1$ equations above can be reduced to:

$$
\begin{aligned}
& \left\|U^{\dagger} U\right\|^{2} \approx \frac{1}{\left(1+\xi^{2}\right)^{2}}+2, \\
& \left\|U^{\dagger} V\right\|^{2} \approx \frac{\xi^{2}}{\left(1+\xi^{2}\right)^{2}}, \\
& \left\|V^{\dagger} V\right\|^{2} \approx \frac{\xi^{4}}{\left(1+\xi^{2}\right)^{2}},
\end{aligned}
$$

where $\xi=\sqrt{m_{v} / m_{N}} \sqrt{\lambda}$. Expressions in Eq. (37) can be regarded as scale relations for norms with respect to a dimensionless scale parameter $\xi$. Again the first identity is closely related to $\operatorname{det}\left(U^{\dagger} U\right) \approx 1 /\left(1+\xi^{2}\right)$ measuring PMNS matrix unitarity. If $R$ matrix depends only on one $\theta$ angle, we find $\lambda=\exp (2|\theta|)$. In other cases $\lambda$ depends on all parameters of $R$ matrix, however if $R$ matrix is parametrized in one of specific ways, $\lambda$ depends only on $\theta_{k}$ angles and not on $\phi_{k}$ angles. More details in Appendix B. Dependence of norms with respect to $\theta_{k}$ and $\phi_{k}$ parameters are shown in Figs. 1 and 2.

\subsection{Extracting neutrino parameters}

Elements of the $O_{L}^{\dagger} U$ matrix contain information about $N_{k}^{M}$ and $v_{j}^{M}$ neutrino masses, $R$ matrix and $S$ matrix elements. In the case $n_{v}=n_{N}$ with fixed $D_{v}$ one can simply obtain $R$ and $D_{N}$ by properly diagonalizing the left-hand side of

$D_{\nu}^{-1 / 2}\left[\left(U^{\dagger} U\right)^{-1}-I\right] D_{\nu}^{-1 / 2}=R\left(-D_{N}\right)^{-1} R^{\dagger}$,

where $U^{\dagger} U=\left(O_{L}^{\dagger} U\right)^{\dagger}\left(O_{L}^{\dagger} U\right)$, since $O_{L}$ is unitary. In case $n_{v}>n_{N}$ projectors $P$ on both sides should be added, but if $n_{N}>n_{v}$ the diagonalization becomes meaningless, since the $S$ matrix appears in various places on the right-hand side of the equation.

Processes involving neutrinos, but not charged leptons in the initial and final state usually do not depend on matrices $O_{L}^{\dagger} U$ and $O_{L}^{\dagger} V$, since masses of charged leptons (in loops) are small compared to the masses of $W$ and $Z$ bosons. Therefore observables in these processes depend only on neutrino masses, $R$ matrix elements and if $n_{N}>n_{v}$ also on $\tilde{S}$ matrix elements. In the heavy $N_{k}$ and pseudo-Dirac neutrino limits such processes are sensitive to (small) non-unitary corrections to the PMNS matrix, but not to PMNS matrix elements themselves. As explained above, outside of both limits the precise relation between the experimentally measured PMNS matrix elements and neutrino parameters is highly nontrivial and thus more difficult to interpret.

\section{Neutral meson decays to two neutrinos and (un)resolved photons}

In this section we consider the decays of pseudoscalar mesons $(P)$ to neutrinos and possibly additional photons $(\gamma)$, as prospective venues to constrain low energy neutrino parameters. We consider both signatures of $P \rightarrow E_{\text {miss }}$ as well as $P \rightarrow E_{\text {miss }} \gamma$, where $E_{\text {miss }}$ is an energy imbalance registered in the particle detector. Within our theoretical setup and assuming a $4 \pi$ detector coverage with finite EM energy resolution, the decay products which contribute to $P \rightarrow E_{\text {miss }}$ include stable enough neutrinos as well as unresolved (soft) photons

$$
\begin{aligned}
& \mathcal{B r}\left(P \rightarrow E_{\text {miss }}\right)=\operatorname{Br}(P \rightarrow \nu v)+\mathcal{B r}\left(P \rightarrow v v \gamma^{*}\right) \\
& \quad+\mathcal{B r}(P \rightarrow \nu v v v)+\cdots,
\end{aligned}
$$

where the dots denote additional multibody decay channels which are further suppressed. The energy of photons present in the final state $\left(\gamma^{*}\right)$, should be less than the energy resolution $E_{0}$ of the EM detector. According to Refs. [36] and [37] the EM calorimeter of Belle II has the energy resolution in the range of $20-50 \mathrm{MeV}$. For concreteness, we use the value $E_{0}=50 \mathrm{MeV}$ throughout this paper. Light neutrinos are clearly invisible to the detector, while heavy neutrinos may decay into lighter and observable decay products before they escape. Thus in general non-trivial conditions which depend on neutrino parameters are imposed on the branching ratio $\operatorname{Br}\left(P \rightarrow E_{\text {miss }}(\gamma)\right)$. We aim to study this dependence and in particular to estimate theoretical upper bounds on $\operatorname{Br}\left(P \rightarrow E_{\text {miss }}(\gamma)\right)$ based on the relevant experimental constraints. To do so, we first briefly discuss the basic properties of $P \rightarrow \nu v$ and $P \rightarrow \nu v \gamma$ decays in the following framework. ${ }^{5}$ We use the Majorana neutrino model with $n_{v}=n_{N}=3$. Details about the model and all relevant equations to reproduce the results are described in Appendix A. We use the following compact notation

$v_{C}=\left\{\begin{array}{l}v_{C}^{M}: C \in\{1,2,3\}, \\ N_{C-3}^{M}: C \in\{4, \ldots, 3+n\},\end{array}\right.$

along with the $\mathcal{U}$ matrix to estimate the relevant branching ratios. ${ }^{6}$ Numerical results and plots in this section are cal-

\footnotetext{
5 As we discuss in detail in Sect. 3.2, the $P \rightarrow v v v v$ contributions are always negligible in our framework.

${ }^{6}$ We note in passing that observables which do not depend on $U^{\dagger} O_{L}$ and $V^{\dagger} O_{L}$ matrix elements can contain $\mathcal{U}_{C D}$ in two forms: $\left|\mathcal{U}_{C D}\right|^{2}$
} 
Fig. 1 Examples of the norms as functions of the $\theta_{2}$ and $\theta_{3}$ parameters in a model with $n_{v}=n_{N}=3$. The other model parameters are fixed as $\theta_{1}=0$ and $\phi_{k}=0$ for all $k$ (implying $\theta_{k} \rightarrow-\theta_{k}$ for each $k$ ). Light neutrino masses are set to satisfy with normal mass hierarchy [35] and $m_{v_{2}} / m_{v_{1}}=2$. The $N_{k}^{M}$ neutrino masses are set to $m_{N}=1 \mathrm{GeV}$ (upper plots) and $m_{N_{1}}=1 \mathrm{MeV}, m_{N_{2}}=1 \mathrm{GeV}$ and $m_{N_{3}}=100 \mathrm{GeV}$ (lower plots) $\left\|U^{\dagger} U\right\|,\left\|U^{\dagger} V\right\|$ and $\left\|V^{\dagger} V\right\|$ present experimental constraints
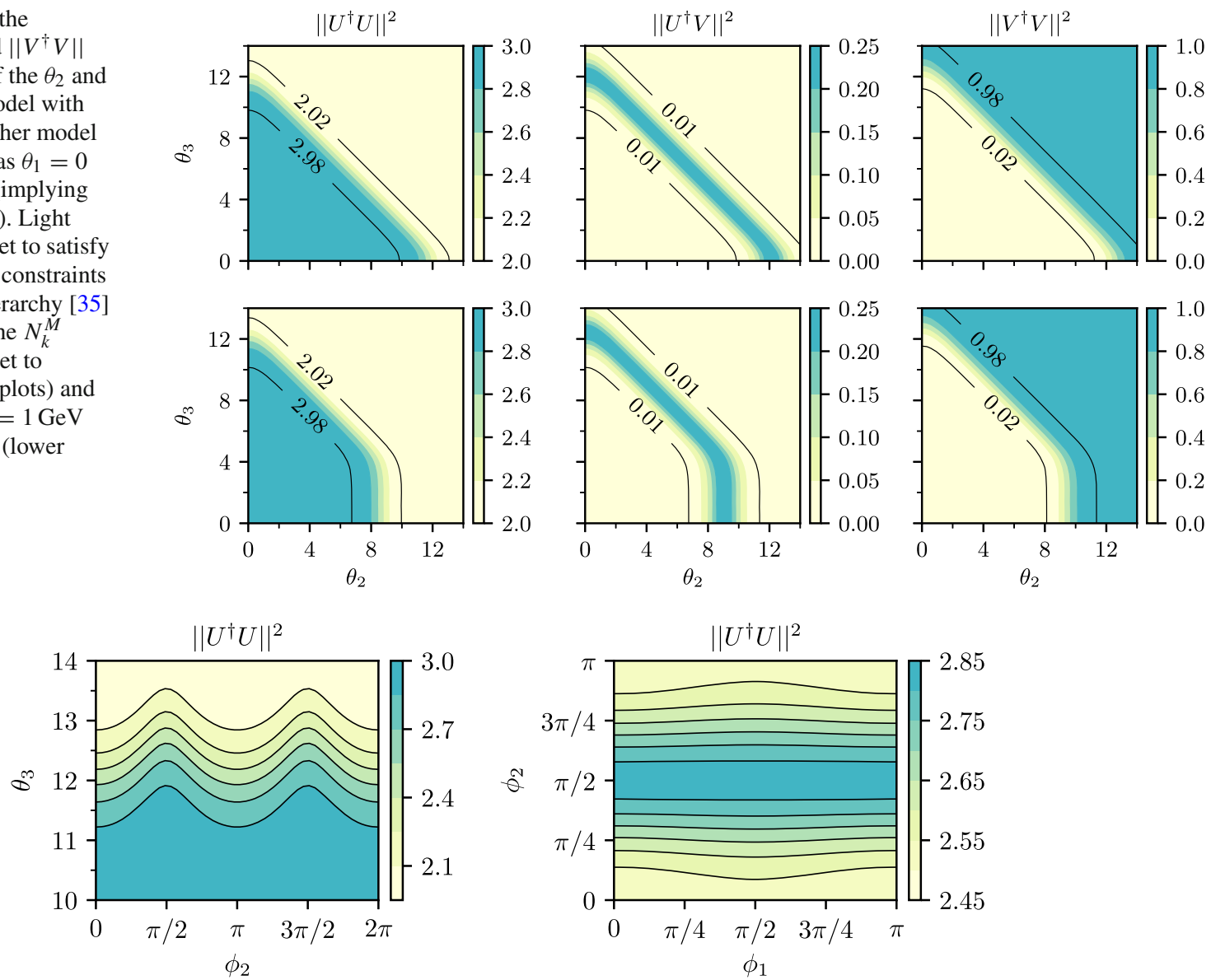

Fig. 2 The $\left\|U^{\dagger} U\right\|$ norm as a function of $\theta_{3}$ and $\phi_{2}$ at $\phi_{1}=0$ (left), as well as $\phi_{2}$ and $\phi_{1}$ at $\theta_{3}=12$ (right), in a $n_{v}=n_{N}=3$ scenario with $m_{N_{1}}=1 \mathrm{MeV}, m_{N_{2}}=1 \mathrm{GeV}$ and $m_{N_{3}}=100 \mathrm{GeV}$. Other $\theta_{j}$ and

culated using expressions and numerical inputs described in Appendix A. For light neutrino masses $m_{v_{j}}$ we impose experimental constraints from [35]. For concreteness, in cases where observables significantly depend on light neutrino masses, we assume a normal mass hierarchy with $m_{v_{2}} / m_{v_{1}}=2$.

\section{$3.1 P \rightarrow v v$}

The $P \rightarrow v_{C} v_{D}$ decay is helicity suppressed and therefore highly sensitive to neutrino masses as can be seen from Eq. (A9). Assume for the moment that $U$ and $R$ matrices are purely real or have a negligibly small imaginary part. Then the dependence of the decay width on $\mathcal{U}$ matrix elements can be factorized such that $\tilde{\mathcal{B}} r\left(P \rightarrow v_{C} v_{D}\right) \equiv \mathcal{B} r(P \rightarrow$

$\left.\overline{\text { and/or } \operatorname{Re}\left(\mathcal{U}_{C D}^{2}\right.}\right)$. Both are unaffected by the change $\mathcal{U}_{C D} \rightarrow \mathcal{U}_{C D}^{*}$. In the case $n_{N} \leq n_{v}$, this implies a symmetry $\theta \rightarrow-\theta$, since $R\left(\phi_{1}, \phi_{2}, \phi_{3}, \theta_{1}, \theta_{2}, \theta_{3}\right)^{*}=R\left(\phi_{1}, \phi_{2}, \phi_{3},-\theta_{1},-\theta_{2},-\theta_{3}\right)$. Exchanging $\theta_{k}$ with $-\theta_{k}$ for a single $k$ is in general not a symmetry of observables.

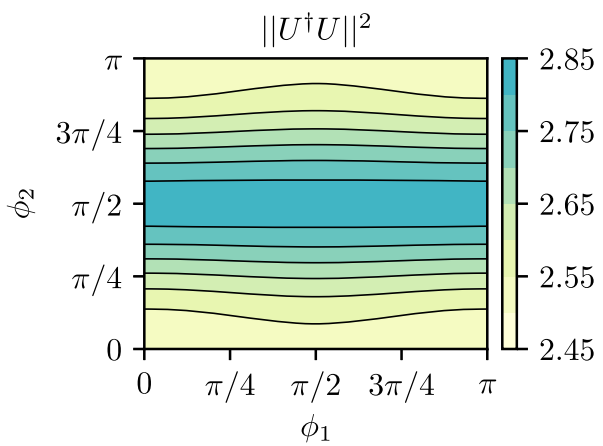

$\phi_{j}$ parameters are set to zero. Light neutrino masses are set to satisfy present experimental constraints with normal mass hierarchy [35] and $m_{v_{2}} / m_{v_{1}}=2$

$\left.v_{C} v_{D}\right) /\left|\mathcal{U}_{C D}\right|^{2}$ becomes independent of $\left|\mathcal{U}_{C D}\right|$ and its dependence on neutrino masses $m_{v_{C}}$ and $m_{v_{D}}$ is shown in Fig. 3.

Note the different kinematical behavior of $P \rightarrow N_{k}^{M} N_{k^{\prime}}^{M}$ and $P \rightarrow v_{j}^{M} N_{k}^{M}$ due to the the sign difference $\operatorname{sgn}[\mathrm{Re}$ $\left.\left(\left(U^{\dagger} V\right)_{j k}^{2}\right)\right]=-\operatorname{sgn}\left[\operatorname{Re}\left(\left(V^{\dagger} V\right)_{k^{\prime} k^{\prime \prime}}^{2}\right)\right]$, see Eq. (A9). Furthermore, assuming that neutrinos are (nearly) degenerate $D_{N} \approx m_{N} I, D_{v} \approx m_{v} I$ with $m_{N} \gg m_{v}$, we can decompose the branching fraction of the $P \rightarrow \nu v$ decay into

$$
\begin{aligned}
\mathcal{B} r(P \rightarrow v v) \approx & \left\|U^{\dagger} U\right\|^{2} \tilde{\mathcal{B}} r\left(P \rightarrow v_{j}^{M} v_{j^{\prime}}^{M}\right) \\
& +2\left\|U^{\dagger} V\right\|^{2} \tilde{\mathcal{B}} r\left(P \rightarrow v_{j}^{M} N_{k}^{M}\right) \\
& +\left\|V^{\dagger} V\right\|^{2} \tilde{\mathcal{B}} r\left(P \rightarrow N_{k}^{M} N_{k^{\prime}}^{M}\right) .
\end{aligned}
$$

In this scenario, norms satisfy scaling relations described in Sect. 2.7. From here it is easy to see that within these assumptions the branching ratio $\operatorname{Br}(P \rightarrow \nu v)$ is largest if $m_{N}$ is of a non-negligible fraction of the $P$ mass and the $\xi$ scale is sufficiently large. This can be seen from Fig. 4 for the case of $B_{s}$ decays. Due to scaling relations similar behavior like in Fig. 4 is expected in case $\theta_{3}$ is exchanged by $\sum_{k} \theta_{k}$. 
Fig. 3 Branching ratio $\mathcal{B} r\left(B_{S} \rightarrow v_{C} v_{D}\right)$ dependence on neutrino masses $m_{v_{C}}$ and $m_{v_{D}}$ assuming $\left(V^{\dagger} V\right)_{k k^{\prime}}$ matrix element is real (left) and $\left(U^{\dagger} V\right)_{j k}$ imaginary (right). See text for details

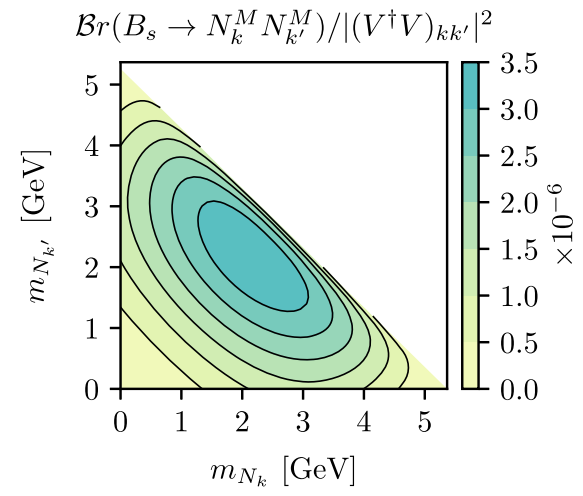

In general the branching fractions $\mathcal{B} r(P \rightarrow \nu v)$ are minimal in the Dirac limit. Precise values depend on the light neutrino masses, but are in any case negligibly small compared to experimental resolution of any currently foreseen experiments [27]. On the other hand, the maximal values of $\tilde{\mathcal{B}} r\left(P \rightarrow v_{C} v_{C^{\prime}}\right)$ are reached at $m_{N_{k}}=m_{N_{k}^{\prime}} \approx 0.4 m_{P}$ for $P \rightarrow N_{k}^{M} N_{k^{\prime}}^{M}$, and at $m_{N_{k}} \approx 0.6 m_{P}$ for $P \rightarrow v_{j}^{M} N_{k}^{M}$. In particular we find $\tilde{\mathcal{B}} r\left(B_{s} \rightarrow N_{k}^{M} N_{k^{\prime}}^{M}\right)_{\max } \simeq 3.5 \times$ $10^{-6}, \tilde{\mathcal{B}} r\left(B_{d} \rightarrow N_{k}^{M} N_{k^{\prime}}^{M}\right)_{\max } \simeq 1.1 \times 10^{-7}$ at $m_{N_{k}}=$ $m_{N_{k^{\prime}}} \simeq 2.2 \mathrm{GeV}$, and $\tilde{\mathcal{B}} r\left(B_{S} \rightarrow v_{j}^{M} N_{k}^{M}\right)_{\max } \simeq 1.3 \times 10^{-6}$, $\tilde{\mathcal{B}} r\left(B_{d} \rightarrow v_{j}^{M} N_{k}^{M}\right)_{\max } \simeq 4.2 \times 10^{-8}$ at $m_{N_{k}} \simeq 3 \mathrm{GeV}$. The maximal values of $\operatorname{Br}(P \rightarrow v v)$, however, also crucially depend on the experimentally allowed values of $\mathcal{U}$ matrix elements. It turns out that currently the constraints are mildest for $m_{N_{k}}$ in the range of a few $\mathrm{GeV}$ [38], in particular, there $\left|\left(U^{\dagger} V\right)_{j k}\right|^{2}<5 \times 10^{-5}$ as reported by the DELPHI collaboration [39]. This bound implies $\xi \ll 1$ and in turn $\left\|V^{\dagger} V\right\| \ll\left\|U^{\dagger} V\right\|$. If we thus assume approximately degenerate $N_{k}$ with masses $m_{N_{k}} \simeq 0.6 M_{B} \sim 3 \mathrm{GeV}$, and all $\left(U^{\dagger} V\right)_{j k}$ matrix elements saturating the current experimental bound in that mass region we obtain

$$
\begin{aligned}
& \mathcal{B} r\left(B_{s} \rightarrow v v\right) \\
& \quad \simeq 2\left\|U^{\dagger} V\right\|^{2} \tilde{\mathcal{B}} r\left(B_{s} \rightarrow v_{j}^{M} N_{k}^{M}\right)_{\max }<1.2 \times 10^{-9}, \\
& \mathcal{B} r\left(B_{d} \rightarrow v v\right) \quad<3.8 \times 10^{-11} .
\end{aligned}
$$

\section{$3.2 P \rightarrow v v \gamma$}

In the limit $m_{C}, m_{D} \ll m_{P}$, the branching ratio $\mathcal{B r}(P \rightarrow$ $\left.v_{C} v_{D} \gamma\right)$ is approximately independent of neutrino masses

$$
\left.\mathcal{B} r\left(P \rightarrow v_{C} v_{D} \gamma\right) \simeq \mathcal{B} r\left(P \rightarrow v_{C} v_{D} \gamma\right)\right|_{m_{C}=m_{D}=0} .
$$

From here the identity $\mathcal{B} r\left(P \rightarrow v_{C} v_{D} \gamma\right) \simeq\left|\mathcal{U}_{C D}\right|^{2} \tilde{\mathcal{B}} r(P \rightarrow$ $\left.v_{C} v_{D} \gamma\right)$, where $\tilde{\mathcal{B}}$ does not depend on $\mathcal{U}_{C D}$, holds. Therefore in the case $\left\|D_{N}\right\| \ll m_{P}$ the branching ratio of the $P \rightarrow v v \gamma$ decay is given by

$$
\begin{aligned}
\mathcal{B} r(P \rightarrow v v \gamma) & =\sum_{C, D} \operatorname{B} r\left(P \rightarrow v_{C} v_{D} \gamma\right) \\
& \left.\simeq \sum_{C, D}\left|\mathcal{U}_{C D}\right|^{2} \tilde{\mathcal{B}} r\left(P \rightarrow v_{C} v_{D} \gamma\right)\right|_{m_{C}=m_{D}=0} \\
& \left.\simeq 3 \tilde{\mathcal{B}} r\left(P \rightarrow v_{C} v_{D} \gamma\right)\right|_{m_{C}=m_{D}=0}
\end{aligned}
$$

which coincides with the SM prediction $\mathcal{B} r(P \rightarrow v v \gamma)^{\mathrm{SM}}$ (with three massless neutrinos). In the last step the identity in Eq. (32) was used. The $\tilde{\mathcal{B}} r\left(P \rightarrow v_{C} v_{D} \gamma\right)$ function takes lower values if neutrino masses $m_{v_{C}}$ and $m_{v_{D}}$ increase as can be seen from the left-hand side plot in Fig. 5 for the case of the $B_{s} \rightarrow N_{k} N_{k^{\prime}} \gamma$ decay where we plot the photon energy spectrum of the decay (normalized to the $B_{s}$ lifetime and the relevant $|\mathcal{U}|^{2}$ matrix element) as a function of the neutrino mass.

Using properties of the $\left\|U U^{\dagger}\right\|$ norm described in Appedix B one finds that in cases $n_{v}=3$ and $n_{N} \in\{2,3\}$ with $\max \left(D_{v}\right) / \min \left(D_{N}\right) \ll 1$ and $\max \left(D_{v}\right) \ll m_{P}$, the branching ratio of $P \rightarrow v v \gamma$ decay lies in the interval:

$$
\frac{2}{3} \leq \frac{\mathcal{B} r(P \rightarrow \nu v \gamma)}{\mathcal{B} r(P \rightarrow \nu v \gamma)^{\mathrm{SM}}} \leq 1 .
$$

The maximal value of the branching ratio takes place in the SM. By taking $\left\|D_{v}\right\|=\left\|D_{N}\right\| \rightarrow 0$, form factors $F_{A}\left(q^{2}\right)$ and $F_{V}\left(q^{2}\right)$ from the most recent estimate [28] and integrating Eq. (A11) over the whole phase-space, we find the SM predictions for branching ratios $\mathcal{B r}\left(B_{s, d} \rightarrow \nu v \gamma\right)$ to be

$$
\begin{aligned}
& \mathcal{B} r\left(B_{s} \rightarrow v v \gamma\right)_{\mathrm{SM}}=6.2(1.9) \times 10^{-9}, \\
& \mathcal{B} r\left(B_{d} \rightarrow v v \gamma\right)_{\mathrm{SM}}=2.8(8) \times 10^{-10},
\end{aligned}
$$

where the $\mathcal{O}(30 \%)$ uncertainties are dominated by the relevant hadronic form factor estimates, see also Appendix A. As can be seen from Table 2, the most recent form factor inputs lead to somewhat reduced predictions compared to previous estimates.

In addition to the branching ratio, measuring the photon energy spectrum in $P \rightarrow v v \gamma$ decays would in principle 
Fig. 4 The $B_{s} \rightarrow v v$ branching ratio as a function of $\theta_{3}$ and $N_{k}^{M}$ neutrino mass $(m)$ in scenario with degenerate $N_{k}^{M}$ neutrino masses with $R=R\left(\theta_{3}\right)$ (upper plots), and in scenario with $m_{N_{1}}=m_{N_{2}}=2 \mathrm{GeV}$, $m=m_{N_{3}}$ and $R=R\left(\theta_{3}\right)$ (lower plots). In both scenarios parameters $\theta_{1}, \theta_{2}, \phi_{k}$ for all $k$ are zero
Fig. 5 Spectrum of the $B_{s} \rightarrow N_{k}^{M} N_{k^{\prime}}^{M} \gamma$ decay as a function of the photon energy $E_{\gamma}$ and neutrino mass $m$ (left). The branching fraction of the $B_{s} \rightarrow N_{k}^{M} N_{k^{\prime}}^{M} \gamma^{*}$ decay as a function of $m$ and photon threshold energy $E_{0}$ (right). In both plots $m=m_{N_{k}}=m_{N_{k^{\prime}}}$ and matrix element $\left(V^{\dagger} V\right)_{k k^{\prime}}$ is assumed to be real. The red curve marks the average value of the photon energy $\left\langle E_{\gamma}\right\rangle$ in the decay as a function of $m$
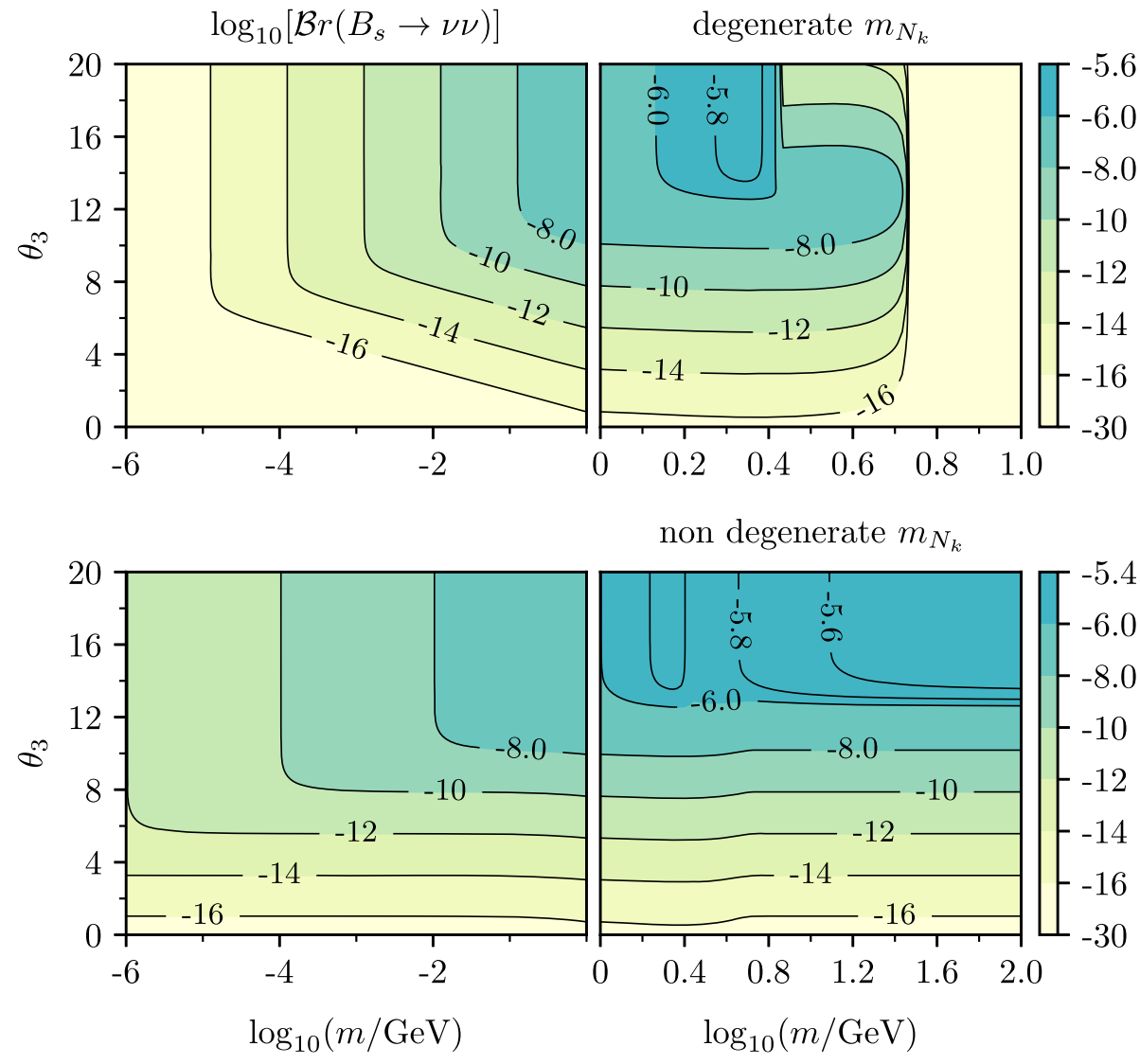

non degenerate $m_{N_{k}}$
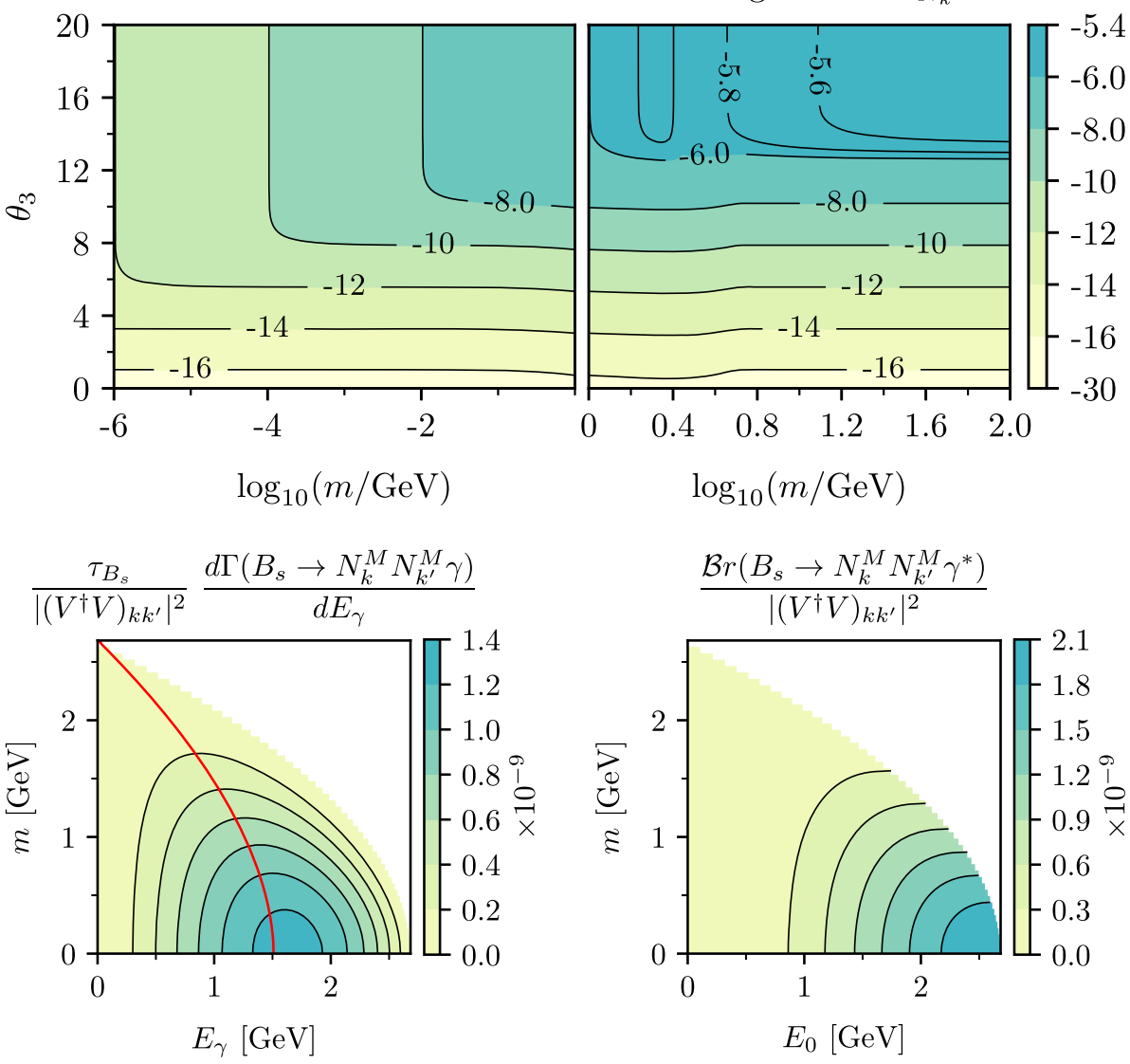

Table 2 SM predictions of branching ratios of decays $\left\{B_{s}, B_{d}\right\} \rightarrow v v \gamma$ decays from other works

\begin{tabular}{llll}
\hline $\mathcal{B} r\left(B_{s} \rightarrow \nu \nu \gamma\right)$ & $\mathcal{B} r\left(B_{d} \rightarrow \nu \nu \gamma\right)$ & Year & References \\
\hline $6.2 \times 10^{-9}$ & $2.8 \times 10^{-10}$ & 2020 & This work \\
$3.68 \times 10^{-8}$ & $1.96 \times 10^{-9}$ & 2010 & {$[27]$} \\
$1.2 \times 10^{-8}$ & Not predicted & 2002 & {$[40]$} \\
$1.8 \times 10^{-8}$ & $2.4 \times 10^{-9}$ & 1996 & {$[41]$} \\
$7.5 \times 10^{-8}$ & $4.2 \times 10^{-9}$ & 1996 & {$[42]$} \\
\hline
\end{tabular}

allow to infer on the mass spectrum of the neutrinos appearing in the final state. In particular, the average photon energy $\left\langle E_{\gamma}\right\rangle$, defined as

$$
\left\langle E_{\gamma}\right\rangle=\frac{1}{\Gamma(P \rightarrow \nu v \gamma)} \int \frac{d \Gamma(P \rightarrow \nu v \gamma)}{d E_{\gamma}} E_{\gamma} d E_{\gamma}
$$

is inversely correlated with final state neutrino masses, as can be seen from the left-hand side plot in Fig. 5.

Assuming neutrinos are completely unobserved, the $P \rightarrow$ $\nu \nu \gamma$ decay contributes also to the invisible $P$ decay width effectively due to the finite resolution of any electromagnetic calorimeter, since photons with energies lower than some threshold energy $E_{0}$ of the detector are not registered. This 
Fig. 6 The branching fraction of the $B_{S} \rightarrow \nu v \gamma^{*}$ decay with a soft photon $\left(E_{\gamma}<E_{0}\right)$ as a function of $\theta_{3}$ and $(m)$ in scenario with degenerate $N_{k}^{M}$ neutrino masses with $R=R\left(\theta_{3}\right)$ (right plot), and in scenario with $m_{N_{1}}=m_{N_{2}}=2 \mathrm{GeV}$, $m=m_{N_{3}}$ and $R=R\left(\theta_{3}\right)$ (left plot). In both scenarios parameters $\theta_{1}, \theta_{2}, \phi_{k}$ for all $k$ are zero

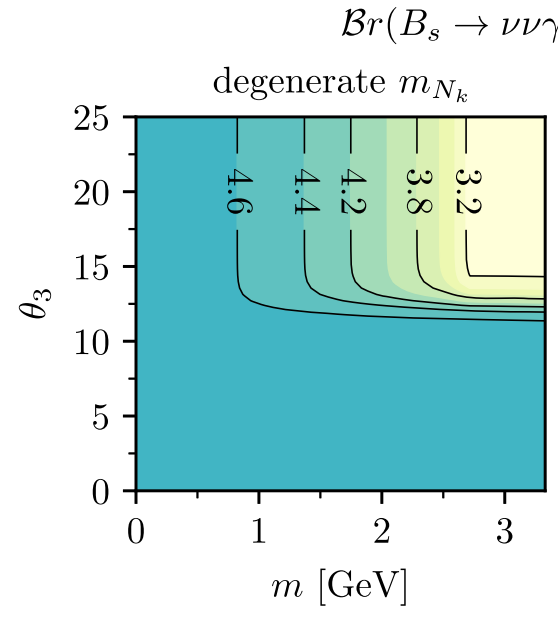

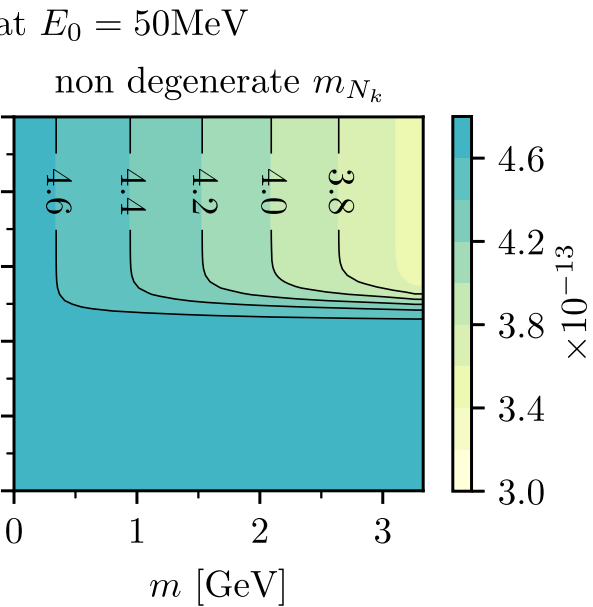

contribution is simply given by

$\mathcal{B} r\left(P \rightarrow \nu v \gamma^{*}\right)=\tau_{P} \int_{0}^{E_{0}} \frac{d \Gamma(P \rightarrow \nu v \gamma)}{d E_{\gamma}} d E_{\gamma}$

where the other integrals are performed over the whole available phase space. On the right-hand side plot in Fig. 5 we show the threshold energy and neutrino mass dependence of the $P \rightarrow v v \gamma^{*}$ decay branching fraction for the case $B_{s} \rightarrow N_{k}^{M} N_{k^{\prime}}^{M} \gamma^{*}$. Specifically, in the SM and for a threshold energy of $E_{0}=50 \mathrm{MeV}$ we obtain the predictions $\operatorname{Br}\left(B_{S} \rightarrow v v \gamma^{*}\right)_{\mathrm{SM}}^{E_{0}=50 \mathrm{MeV}}=4.7 \times 10^{-13}$ and $\operatorname{Br}\left(B_{d} \rightarrow v v \gamma^{*}\right)_{\mathrm{SM}}^{E_{0}=50 \mathrm{MeV}}=2.5 \times 10^{-14}$. We show in Fig. 6 a typical dependence of the $B_{s} \rightarrow v v \gamma^{*}$ branching fraction on the neutrino mixing parameters in two scenarios for $N_{k}^{M}$ neutrino masses, $E_{0}=50 \mathrm{MeV}$ and with $R=R\left(\theta_{3}\right), \theta_{1}=\theta_{2}=0$. In comparison to Fig. 4 we observe that these contributions to $B_{S} \rightarrow E_{\text {miss }}$ are generically still more than three orders of magnitude smaller than the current upper limit on $B_{S} \rightarrow \nu \nu$ and thus completely subleading. However, at the same time, $P \rightarrow v v \gamma^{*}$ are always much bigger than $P \rightarrow \nu \nu \nu \nu$ [29] and are thus expected to dominate $P \rightarrow E_{\text {miss }}$ in the (pseudo)Dirac neutrino limit. In addition, $P \rightarrow v v \gamma$ might contribute effectively to $P \rightarrow E_{\text {miss }}$ also due to other detector effects, such as non-perfect $4 \pi$ coverage. Such contributions are however difficult to model without a detailed knowledge of the detector components and geometry and we leave such a study to the experimental collaborations.

\section{Conclusions}

In this work we have reconsidered Majorana neutrino mass models and derived a model-independent general parametrization of neutrino mass matrices with physically interpretable and irreducible set of parameters. The parametrization is valid for any number of left-handed (as in SM) and right-handed (gauge singlet Majorana) neutrinos and for all mass hierarchies. In particular, in the heavy Majorana neutrino limit we recover the standard Casas-Ibara parametrization [18], while the parametrization nicely interpolates also through the (pseudo)Dirac neutrino limit.

We have applied the new parametrization to the study of $P \rightarrow \nu v$ and $P \rightarrow \nu v \gamma$ decays within the SM extended by $n_{N}=3$ additional singlet neutrinos. ${ }^{7}$ Along the way we have updated the SM predictions for the branching ratios of $B_{s, d} \rightarrow v v \gamma$ decays and found almost an order of magnitude smaller values compared to previous estimates, mainly due to a recent reevaluation of the relevant hadronic form factors.

Finally, we have discussed the sensitivity of the $B_{s, d} \rightarrow$ $E_{\text {miss }}(\gamma)$ decays to neutrino mass and mixing parameters. In the case of $B_{s, d} \rightarrow E_{\text {miss }}$, for typical EM calorimeter threshold energies and assuming $4 \pi$ coverage, the dominant contribution could still come from $B_{s, d} \rightarrow \nu v$ decays where one of the final state neutrinos is predominantly a SM gauge singlet of mass of the order a few GeV. However, the maximum allowed branching ratios, given by the current experimental bounds on the relevant neutrino mixing matrices, are at least four orders of magnitude below the direct limits from the B factories. It remains to be seen if Belle II can reach the required sensitivity to constrain the parameter space of neutrino mass models in this interesting region.

In the case of $B_{s, d} \rightarrow E_{\text {miss }} \gamma$ decays, additional light neutrinos in the final state could affect both the branching ratios as well as the photon energy spectra and thus in principle allow to extract information on the neutrino mass parameters. Unfortunately, however, possible deviations from SM predictions (i.e. the limit of massless neutrinos) are theoretically constrained and at most comparable to current uncer-

\footnotetext{
7 The parametrization can trivially be applied to other neutral current mediated processes involving pairs of neutrinos. In the case of charged current mediated processes commonly used to constrain the PMNS matrix (see e.g. Ref. [43]), or in searches for massive Majorana neutrinos with lepton number violating signatures $[33,44]$, one instead needs to consider additional dependence on the unitary $O_{L}$ matrix.
} 
tainties due to the limited knowledge of the relevant form factors. Any relevant experimental sensitivity to neutrino mass parameters is thus conditional upon an improved understanding of the relevant hadronic parameters, which could possibly come from future Lattice QCD studies (see Refs. [45,46] for current prospects).

Acknowledgements We are grateful to Klemen Šivic for his help in the derivation of the lower bound on the $\left\|U U^{\dagger}\right\|$ norm, and to Miha Nemevšek for his comments on the manuscript. JFK acknowledges the financial support from the Slovenian Research Agency (research core funding No. P1-0035 and J1-8137).

Data Availability Statement This manuscript has no associated data or the data will not be deposited. [Authors' comment: The results presented in the paper are theoretical and no data beyond that shown in the figures (readily derivable using analytic formulae and inputs presented in the paper) has been generated.]

Open Access This article is licensed under a Creative Commons Attribution 4.0 International License, which permits use, sharing, adaptation, distribution and reproduction in any medium or format, as long as you give appropriate credit to the original author(s) and the source, provide a link to the Creative Commons licence, and indicate if changes were made. The images or other third party material in this article are included in the article's Creative Commons licence, unless indicated otherwise in a credit line to the material. If material is not included in the article's Creative Commons licence and your intended use is not permitted by statutory regulation or exceeds the permitted use, you will need to obtain permission directly from the copyright holder. To view a copy of this licence, visit http://creativecomm ons.org/licenses/by/4.0/.

Funded by SCOAP ${ }^{3}$.

\section{Appendix A: Calculation of $P \rightarrow v v$ and $P \rightarrow v v \gamma$ decay widths}

Below we summarize our calculation of the $B_{s, d} \rightarrow v v(\gamma)$ decay widths as discussed in the main text. We consider the physically relevant scenario with $n_{v}=3$ neutrinos $v_{j}^{M}$ and $n_{N}$ neutrinos $N_{k}^{M}$. The Majorana fields are defined in a way such that the Dirac limit can be approached analytically with both $D_{v}$ and $D_{N}$ matrices being positive semi-definite. For other choices of the relecant phase factors one should properly redefine the mixing matrix $\mathcal{U}$. Note that our calculation can be applied also to the corresponding $K, D$ meson decays, with suitable quark flavor replacements.

We calculate the decay widths $B_{s, d} \rightarrow \nu v(\gamma)$ using the relevant effective weak Lagrangian $[47,48]$

$\mathcal{L}_{\text {eff }}=\frac{4 G_{F} \alpha_{\mathrm{em}}}{2 \sqrt{2} \pi \sin ^{2} \theta_{W}} \sum_{q=s, d} V_{t q}^{*} V_{t b} X\left(x_{t}\right)\left[\bar{b} \gamma_{\mu} P_{L} q\right] \sum_{C, D=1}^{3+n} J_{C D}^{\mu}$, where the leptonic current $J_{C D}^{\mu}$ is given by

$$
\begin{aligned}
J_{C D}^{\mu} & =\left\langle v_{C}\left(\mathbf{p}_{C}\right), v_{D}\left(\mathbf{p}_{D}\right)\left|\sum_{a=1}^{3} \overline{v_{a L}} \gamma^{\mu} v_{a L}\right| 0\right\rangle \mathrm{e}^{-\mathrm{i}\left(p_{C}+p_{D}\right) x}, \\
& =-\mathcal{U}_{C D}\left[\bar{u}_{C} \gamma^{\mu} P_{L} v_{D}\right]+\mathcal{U}_{C D}^{*}\left[\bar{u}_{D} \gamma^{\mu} P_{L} v_{C}\right] .
\end{aligned}
$$

The relevant loop function $X\left(x_{t}\right)$ can be written as

$X\left(x_{t}, x_{\mu}\right)=X_{0}\left(x_{t}\right)+\frac{\alpha_{s}(\mu)}{4 \pi} X_{1}\left(x_{t}, x_{\mu}\right)$,

where $X_{0}\left(x_{t}\right)$ is the Inami-Lim function [47]

$X_{0}\left(x_{t}\right)=\frac{x_{t}}{8}\left[\frac{x_{t}+2}{x_{t}-1}+\frac{3\left(x_{t}-2\right)}{\left(x_{t}-1\right)^{2}} \ln x_{t}\right]$,

and the leading QCD corrections are parametrized by $X_{1}$ whose explicit expression can be found in Refs. [48,49]. Here $x_{\mu}=\mu^{2} / M_{W}^{2}, x_{t}=m_{t}^{2} / M_{W}^{2}$ and the $\overline{\mathrm{MS}}$ QCD renormalization scheme is assumed throughout. In the following we compress the common constant prefactors entering the Lagrangian into

$C \equiv \frac{G_{F} \alpha_{\mathrm{em}}}{2 \sqrt{2} \pi \sin ^{2} \theta_{W}} V_{t q}^{*} V_{t b} X\left(x_{t}\right)$.

Above and in the following we have suppressed the light flavor $(q=s, d)$ indices where the identification of the relevant $B_{(q)}$ meson flavor is unambiguous.

For the $P \rightarrow v v$ decay we parametrize the relevant hadronic matrix elements in the standard way

$$
\begin{aligned}
\left\langle 0\left|\bar{b} \gamma^{\mu} q\right| P(p)\right\rangle & =0, \\
\left\langle 0\left|\bar{b} \gamma^{\mu} \gamma^{5} q\right| P(p)\right\rangle & =\mathrm{i} f_{P} p^{\mu},
\end{aligned}
$$

where $f_{P}$ is the relevant $P=B_{s, d}$ meson decay constant. In particular, we use $f_{B_{s}}=224 \mathrm{MeV}$ and $f_{B_{d}}=186 \mathrm{MeV}$ from Ref. [50].

In the case of the radiative decay, only the emission of photons from the hadronic part is relevant and is parametrized by the relevant radiative form factors

$$
\begin{aligned}
\left\langle\gamma(k)\left|\bar{b} \gamma_{\mu} q\right| P(k+q)\right\rangle & =e \epsilon_{\mu \nu \rho \sigma} \epsilon^{* v} q^{\rho} k^{\sigma} \frac{F_{V}\left(q^{2}\right)}{m_{P}}, \\
\left\langle\gamma(k)\left|\bar{b} \gamma_{\mu} \gamma_{5} q\right| P(k+q)\right\rangle & =-\mathrm{i} e\left[\epsilon_{\mu}^{*}(k q)-\left(\epsilon^{*} q\right) k_{\mu}\right] \frac{F_{A}\left(q^{2}\right)}{m_{P}} .
\end{aligned}
$$

Here $q=p_{C}+p_{D}=p-k$ and $q^{2}=m_{P}^{2}-2 m_{P} E_{\gamma}$. For the axial (A) and vectorial (V) form factors $F_{A}\left(q^{2}\right)$ and $F_{V}\left(q^{2}\right)$ 
we take the most recent estimate [28] parametrized by

$F_{X}\left(q^{2}\right)=\frac{F(0)}{\left(1-q^{2} / M_{R}^{2}\right)\left[1-\sigma_{1}\left(q^{2} / M_{R}^{2}\right)+\sigma_{2}\left(q^{2} / M_{R}^{2}\right)^{2}\right]}$,

where $F(0), \sigma_{1}, \sigma_{2}$ and $M_{R}$ parameters for $P=B_{s, d}$ are given in Ref. [28]. After a quick calcuation one finds the expression for the $P \rightarrow v_{C} v_{D}$ decay width

$$
\begin{aligned}
& \Gamma\left(P \rightarrow v_{C} v_{D}\right)=16|C|^{2} f_{P}^{2}\left\{\frac { 1 } { 2 } | \mathcal { U } _ { C D } | ^ { 2 } \left[m_{P}^{2}\left(m_{C}^{2}+m_{D}^{2}\right)\right.\right. \\
& \left.\quad-\left(m_{C}^{2}-m_{D}^{2}\right)^{2}\right] \\
& \left.\quad+m_{C} m_{D} m_{P}^{2} \operatorname{Re}\left(\mathcal{U}_{C D}^{2}\right)\right\} \frac{Q}{2 m_{P}}
\end{aligned}
$$

where

$$
\begin{aligned}
Q= & \frac{1}{(4 \pi) m_{P}} \sqrt{\left(\frac{m_{P}^{2}+m_{C}^{2}-m_{D}^{2}}{2 m_{P}}\right)^{2}-m_{C}^{2}} \\
& \times\left\{\begin{array}{l}
1 \text { : Dirac, } \\
\frac{1}{2} \text { : Majorana. }
\end{array}\right.
\end{aligned}
$$

In the Dirac limit the process $P \rightarrow v_{j}^{M} N_{j}^{M}$ is forbidden. Similarly, the triply differential $P \rightarrow v_{C} v_{D} \gamma$ decay width (for Majorana neutrinos) is given by

$$
\begin{aligned}
& \frac{d^{3} \Gamma\left(P \rightarrow v_{C} v_{D}\right)}{d E_{\gamma} d E_{C} d \Omega} \\
& =\frac{|C|^{2} \alpha_{\mathrm{em}}}{4 \pi^{3} m_{P}}\left[F_{A}^{2}+F_{V}^{2}\right]\left\{-m_{C} m_{D} E_{\gamma}^{2} \operatorname{Re}\left(\mathcal{U}_{C D}^{2}\right)\right. \\
& \quad+\left|\mathcal{U}_{C D}\right|^{2}\left[\left(k \cdot p_{C}\right)^{2}\right. \\
& \left.\left.\quad-E_{\gamma}\left(2 E_{C}+E_{\gamma}\right)\left(k \cdot p_{C}\right)+E_{\gamma}^{2} E_{C} m_{P}\right]\right\} \\
& \quad \times \theta\left(m_{P}-E_{C}-E_{\gamma}\right) \theta\left(E_{C}-E_{C \min }\right) \theta\left(E_{C \max }-E_{C}\right) .
\end{aligned}
$$

Note that due to Majorana nature of neutrinos the full integral over the solid angle $d \Omega$ gives $2 \pi$ instead of the usual $4 \pi$. From kinematic constraints one furthermore obtains

$$
E_{C_{\min }}^{\max }=\frac{m_{P}-E_{\gamma}}{2} \Delta \pm \frac{1}{2} E_{\gamma} \sqrt{\Delta^{2}-\frac{4 m_{C}^{2}}{m_{P}^{2}-2 m_{P} E_{\gamma}}}
$$

where is $\Delta$ equal to

$$
\Delta=1+\frac{m_{C}^{2}-m_{D}^{2}}{m_{P}^{2}-2 m_{P} E_{\gamma}}
$$

while $k \cdot p_{C}$ is given by

$k \cdot p_{C}=\frac{1}{2}\left(-m_{P}^{2}-m_{C}^{2}+m_{D}^{2}+2 m_{P} E_{\gamma}+2 m_{P} E_{c}\right)$.

Finally, in our numerical results we use $\mu=m_{Z}=$ $91.2 \mathrm{GeV}, \alpha_{\mathrm{em}}=1 / 137, m_{B_{s}}=5.37 \mathrm{GeV}, m_{B_{d}}=$ $5.28 \mathrm{GeV}, m_{W}=80.4 \mathrm{GeV},\left|V_{t b} V_{t s}^{*}\right|=0.0403,\left|V_{t b} V_{t d}^{*}\right|=$ $0.00875, \sin ^{2}\left(\theta_{W}\right)=0.22, \tau_{B_{s}}=1.51 \mathrm{ps}, \tau_{B_{d}}=1.52 \mathrm{ps}$, $\alpha_{s}\left(m_{Z}\right)=0.118$ and $m_{t}\left(m_{Z}\right)=172 \mathrm{GeV}$ [51].

\section{Appendix B: Lower bounds on $\left\|U U^{\dagger}\right\|$}

In this section we formally prove Eq. (45) and discuss additional properties of the $\left\|U U^{\dagger}\right\|$ norm. We assume a model with $n_{v}$ light neutrinos $\left(m_{v_{j}} / m_{P} \ll 1\right)$ and $n_{N}$ heavy neutrinos $\left(m_{v_{j}} / m_{N_{k}} \ll 1\right)$. From properties of $P \rightarrow v v \gamma$ decay follows that inequality:

$\left.\left\|U U^{\dagger}\right\|^{2} \tilde{\mathcal{B}} r\left(P \rightarrow v_{j} v_{j} \gamma\right)\right|_{m_{v_{j}}=0} \leq \mathcal{B} r(P \rightarrow v v \gamma)$,

holds. Moreover from upper limit of the branching ratio $\mathcal{B r}(P \rightarrow \nu \nu \gamma)$ we find:

$\left.\tilde{\mathcal{B}} r\left(P \rightarrow v_{j} v_{j} \gamma\right)\right|_{m_{v_{j}}=0}=\frac{1}{n_{v}} \mathcal{B} r(P \rightarrow v v \gamma)^{\mathrm{SM}}$

This is a direct consequence of Eq. (32). From here, within assumed model we have:

$\frac{\left\|U U^{\dagger}\right\|^{2}}{n_{v}} \leq \frac{\mathcal{B} r(P \rightarrow v v \gamma)}{\mathcal{B} r(P \rightarrow \nu v \gamma)^{\mathrm{SM}}} \leq 1$.

Value of the norm is due to derived parametrization of neutrino matrices directly related to the eigenvalues $\mu_{j}$ of the $Q Q^{\dagger}$ matrix through relation:

$\left\|U^{\dagger} U\right\|^{2}=\sum_{j=1}^{n_{v}} \frac{1}{\left(1+\mu_{j}\right)^{2}}$.

This equation is obtained by diagonalizing $Q Q^{\dagger}$ matrix inside Frobenius norm. For $U V^{\dagger}$ and $V V^{\dagger}$ matrices similar formulas can be found:

$$
\begin{aligned}
\left\|U^{\dagger} V\right\|^{2}= & \left\|V^{\dagger} U\right\|^{2}=\sum_{j=1}^{n_{v}} \frac{\mu_{j}}{\left(1+\mu_{j}\right)^{2}}, \\
\left\|V^{\dagger} V\right\|^{2} & =\sum_{j=1}^{n_{v}} \frac{\mu_{j}^{2}}{\left(1+\mu_{j}\right)^{2}} .
\end{aligned}
$$


We use Eq. (B4) as a starting point to determine theoretical lower bounds on the $\left\|U U^{\dagger}\right\|$ norm for different choices of $n_{v}$ and $n_{N}$.

\subsection{Case $n_{v} \geq n_{N}$}

In scenario with $n_{v} \geq n_{N}$ we use following theorems.

Notation 1 Let $X$ be a $n \times n$ hermitian matrix, then we denote its eigenvalues as $\lambda_{j}(X)$, where $\lambda_{1}(X)<\cdots<\lambda_{n}(X)$.

Definition 1 (Loewner order) Let $A$ and $B$ be hermitian matrices, then $A \leq B$ if and only if $A-B$ is positive semidefinite matrix.

Theorem 1 (Loewner order is compatible with congruence) If $A$ and $B$ are hermitian matrices and $A \leq B$, then for any matrix $X: X A X^{\dagger} \leq X B X^{\dagger}$.

Theorem 2 (Wely's monotonicity theorem, [[52], Corollary 4.3.3]) If $A$ and $B$ are $n \times n$ hermitian matrices with $A \leq B$, then $\lambda_{k}(A) \leq \lambda_{k}(B)$ for each $k=1, . ., n$.

Theorem 3 ([[52], Theorem 1.3.20]) Let A be a $m \times n$ matrix and $B$ be a $n \times m$ matrix with $m \leq n$. Then $B A$ matrix has the same eigenvalues as $B$ A, together with additional $n-m$ eigenvalues equal to 0 .

We denote by $a$ the largest eigenvalue of $D_{v}$ and by $b$ the smallest eigenvalue of $D_{N}$, therefore $D_{N}^{-1} \leq 1 / b I$. Using Theorem 1 we get:

$Q Q^{\dagger}=\left(D_{\nu}^{1 / 2} P R\right) D_{N}^{-1}\left(D_{\nu}^{1 / 2} P R\right)^{\dagger} \leq \frac{1}{b} D_{\nu}^{1 / 2} P R R^{\dagger} P^{\dagger} D_{\nu}^{1 / 2}$

By Theorem 3 we find $\lambda_{j}\left(Q Q^{\dagger}\right)=0$ for $j=1, \ldots, n_{v}-$ $n_{N}$. Next for $j>n_{v}-n_{N}$, we apply Theorems 2,3 and 1 in this order to obtain:

$$
\begin{gathered}
\lambda_{j}\left(Q Q^{\dagger}\right) \leq \frac{1}{b} \lambda_{j}\left(\left(D_{v}^{1 / 2} P R\right)\left(R^{\dagger} P^{\dagger} D_{v}^{1 / 2}\right)\right) \\
\leq \frac{1}{b} \lambda_{j}\left(R^{\dagger} P^{\dagger} D_{v} P R\right) \leq \frac{a}{b} \lambda_{j}\left(R^{\dagger} R\right) .
\end{gathered}
$$

From here, we finally get:

$$
\left\|U^{\dagger} U\right\|^{2} \geq n_{v}-n_{N}+\sum_{k=1}^{n_{N}} \frac{1}{\left[1+\frac{a}{b} \lambda_{k}\left(R R^{\dagger}\right)\right]^{2}} .
$$

From the property $\left(R^{\dagger} R\right)^{-1}=\left(R^{\dagger} R\right)^{*}$ follows directly, that if $\lambda\left(R R^{\dagger}\right)$ is an eigenvalue of the $R^{\dagger} R$ matrix, then $1 / \lambda\left(R^{\dagger} R\right)$ is also eigenvalue of $R^{\dagger} R$ matrix. A consequence of this property is that for any $(2 n+1) \times(2 n+1)$ orthogonal matrix $R$, the $R R^{\dagger}$ matrix has at least one eigenvalue equal to 1 . Therefore, if $R$ is a general $n \times n$ complex orthogonal matrix, then $R^{\dagger} R$ matrix have maximally $\lfloor n / 2\rfloor$ (integer part of $n / 2$ ) eigenvalues which are greater than 1 . This implies:

$\left\|U^{\dagger} U\right\|^{2} \geq n_{v}-\left\lfloor n_{N} / 2\right\rfloor$

Using property:

$\frac{1}{\left(1+\frac{a}{b} \lambda\right)^{2}}+\frac{1}{\left(1+\frac{a}{b} \frac{1}{\lambda}\right)^{2}} \geq \frac{1}{\left(1+\frac{a}{b}\right)^{2}}$

a better lower bound can be obtained:

$\left\|U^{\dagger} U\right\|^{2} \geq n_{v}-n_{N}+\frac{\left\lfloor\frac{n_{N}+1}{2}\right\rfloor}{\left(1+\frac{a}{b}\right)^{2}}$.

Eigenvalues of $R R^{\dagger}$ matrix depend only on $\theta_{k}$ parameters if $R$ matrix is parametrized in the following way:

$R(\underline{\phi}, \underline{\theta})=\prod_{k=1}^{n} R_{k}\left(\phi_{k}\right) \prod_{k=1}^{n} R_{n+k}\left(\theta_{k}\right)$

where $R_{1}, \ldots, R_{n}$ are real orthogonal matrices and therefore unitary. We can absorb them in the process of diagonalizing $R R^{\dagger}$ matrix:

$$
\begin{aligned}
& \operatorname{det}\left(\prod_{k=1}^{n} R_{k}\left(\phi_{k}\right) \prod_{k=1}^{n} R_{n+k}\left(\theta_{k}\right) R_{n+k}^{\dagger}\left(\theta_{k}\right) \prod_{k=1}^{n} R_{k}^{\dagger}\left(\phi_{k}\right)-\lambda I\right) \\
& =\operatorname{det}\left(\prod_{k=1}^{n} R_{n+k}\left(\theta_{k}\right) R_{n+k}^{\dagger}\left(\theta_{k}\right)-\lambda I\right) .
\end{aligned}
$$

From here, eigenvalues of $R R^{\dagger}$ matrix must depend only on $\theta_{k}$ parameters.

\subsection{Case $n_{v}<n_{N}$}

In scenario with $n_{v}<n_{N}$ is more difficult to obtain eigenvalues of $Q Q^{\dagger}$ matrix, since $S$ matrix is present in it. In case $n_{N} \geq 2 n_{v}$ the lowest bound on $\left\|U U^{\dagger}\right\|$ norm is 0 . This can be proven using $R=I_{n_{v} \times n_{v}}, D_{v}=a I_{n_{v} \times n_{v}}$, $S=\left[I_{n_{v} \times n_{v}}, \mathrm{i} x I_{n_{v} \times n_{v}}, 0_{\left(n_{N}-2 n_{v}\right) \times\left(n_{N}-2 n_{v}\right)}\right]$, where $x \in \mathcal{R}$ and:

$D_{N}=\left(\begin{array}{ccc}b I_{n_{v} \times n_{v}} & 0 & 0 \\ 0 & c I_{n_{v} \times n_{v}} & 0 \\ 0 & 0 & C_{\left(n_{N}-2 n_{v}\right) \times\left(n_{N}-2 n_{v}\right)}\end{array}\right)$

where $C$ is a positive semi-definite diagonal matrix. From here one gets:

$Q Q^{\dagger}=a \frac{1+x^{2}}{\left|b-c x^{2}\right|} I_{n_{v} \times n_{v}}$ 
For $x \neq \pm \sqrt{b / c}$ matrix $S D_{N} S^{T}$ is invertible. When $x$ approaches to $\sqrt{b / c}$ eigenvalues of $Q Q^{\dagger}$ matrix become very large and therefore the lowest value for the $\left\|U U^{\dagger}\right\|$ norm is 0 .

\section{References}

1. Y. Fukuda et al., Evidence for oscillation of atmospheric neutrinos. Phys. Rev. Lett. 81, 1562-1567 (1998). https://doi.org/10.1103/ PhysRevLett.81.1562. arXiv:hep-ex/9807003

2. M. Gell-Mann, P. Ramond, R. Slansky, Complex spinors and unified theories. Conf. Proc. C 790927, 315-321 (1979). arXiv: 1306.4669

3. P. Minkowski, $\mu \rightarrow e \gamma$ at a Rate of One Out of $10^{9}$ Muon Decays? Phys. Lett. B 67, 421-428 (1977). https://doi.org/10. 1016/0370-2693(77)90435-X

4. R.N. Mohapatra, G. Senjanovic, Neutrino Mass and Spontaneous Parity Nonconservation. Phys. Rev. Lett. 44, 912 (1980). https:// doi.org/10.1103/PhysRevLett.44.912

5. M. Magg, C. Wetterich, Neutrino mass problem and Gauge hierarchy. Phys. Lett. B 94, 61-64 (1980). https://doi.org/10.1016/ 0370-2693(80)90825-4

6. G. Lazarides, Q. Shafi, C. Wetterich, Proton lifetime and fermion masses in an SO(10) model. Nucl. Phys. B 181, 287-300 (1981). https://doi.org/10.1016/0550-3213(81)90354-0

7. A. Ibarra, E. Molinaro, S. Petcov, TeV scale see-saw mechanisms of neutrino mass generation, the Majorana nature of the heavy singlet neutrinos and $(\beta \beta)_{0 v}$-decay. JHEP 09, 108 (2010). https://doi.org/ 10.1007/JHEP09(2010)108. arXiv:1007.2378

8. D. Aristizabal Sierra, A. Degee, J. Kamenik, Minimal lepton flavor violating realizations of minimal seesaw models. JHEP 07, 135 (2012). https://doi.org/10.1007/JHEP07(2012)135. arXiv: 1205.5547

9. K. Abazajian, G.M. Fuller, W.H. Tucker, Direct detection of warm dark matter in the X-ray. Astrophys. J. 562, 593-604 (2001). https:// doi.org/10.1086/323867. arXiv:astro-ph/0106002

10. M. Viel, J. Lesgourgues, M.G. Haehnelt, S. Matarrese, A. Riotto, Constraining warm dark matter candidates including sterile neutrinos and light gravitinos with WMAP and the Lyman-alpha forest. Phys. Rev. D 71, 063534 (2005). https://doi.org/10.1103/ PhysRevD.71.063534. arXiv:astro-ph/0501562

11. S. Dodelson, L.M. Widrow, Sterile-neutrinos as dark matter. Phys. Rev. Lett. 72, 17-20 (1994). https://doi.org/10.1103/PhysRevLett. 72.17. arXiv:hep-ph/9303287

12. K. Abazajian, G.M. Fuller, M. Patel, Sterile neutrino hot, warm, and cold dark matter. Phys. Rev. D 64, 023501 (2001). https://doi. org/10.1103/PhysRevD.64.023501. arXiv:astro-ph/0101524

13. X.-D. Shi, G.M. Fuller, A New dark matter candidate: Nonthermal sterile neutrinos. Phys. Rev. Lett. 82, 2832-2835 (1999). https:// doi.org/10.1103/PhysRevLett.82.2832. arXiv:astro-ph/9810076

14. A. Dolgov, S. Hansen, Massive sterile neutrinos as warm dark matter. Astropart. Phys. 16, 339-344 (2002). https://doi.org/10.1016/ S0927-6505(01)00115-3. arXiv:hep-ph/0009083

15. T. Asaka, S. Blanchet, M. Shaposhnikov, The nuMSM, dark matter and neutrino masses. Phys. Lett. B 631, 151-156 (2005). https:// doi.org/10.1016/j.physletb.2005.09.070. arXiv:hep-ph/0503065

16. T. Asaka, M. Shaposhnikov, The $v$ MSM, dark matter and baryon asymmetry of the universe. Phys. Lett. B 620, 17-26 (2005). https:// doi.org/10.1016/j.physletb.2005.06.020. arXiv:hep-ph/0505013

17. K.N. Abazajian, et al., Light sterile neutrinos: A white paper (4 2012). arXiv:1204.5379
18. J.A. Casas, A. Ibarra, Oscillating neutrinos and muon $\longrightarrow$ e, gamma. Nucl. Phys. B 618, 171-204 (2001). https://doi.org/10. 1016/S0550-3213(01)00475-8. arXiv:hep-ph/0103065

19. A. Donini, P. Hernandez, J. Lopez-Pavon, M. Maltoni, T. Schwetz, The minimal $3+2$ neutrino model versus oscillation anomalies. JHEP 07, 161 (2012). https://doi.org/10.1007/JHEP07(2012)161. arXiv: 1205.5230

20. P .D. Bolton, F .F. Deppisch, P. Bhupal Dev, Neutrinoless double beta decay versus other probes of heavy sterile neutrinos. JHEP 03, 170 (2020). https://doi.org/10.1007/JHEP03(2020)170. arXiv: 1912.03058

21. M. Blennow, E. Fernandez-Martinez, Parametrization of seesaw models and light sterile neutrinos. Phys. Lett. B 704, 223-229 (2011). https://doi.org/10.1016/j.physletb.2011.09.028. arXiv:1107.3992

22. P. Hernandez, M. Kekic, J. Lopez-Pavon, $N_{\text {eff }}$ in low-scale seesaw models versus the lightest neutrino mass. Phys. Rev. D 90(6), 065033 (2014). https://doi.org/10.1103/PhysRevD.90. 065033. arXiv: 1406.2961

23. N.R. Agostinho, G. Branco, P.M.F. Pereira, M. Rebelo, J. SilvaMarcos, Can one have significant deviations from leptonic 3 $\times 3$ unitarity in the framework of type I seesaw mechanism? Eur. Phys. J. C 78(11), 895 (2018). https://doi.org/10.1140/epjc/ s10052-018-6347-2. arXiv:1711.06229

24. I. Cordero-Carrión, M. Hirsch, A. Vicente, General parametrization of Majorana neutrino mass models (2019). arXiv:1912.08858

25. N. Arkani-Hamed, T. Cohen, R.T. D’Agnolo, A. Hook, H.D. Kim, D. Pinner, Solving the hierarchy problem at reheating with a large number of degrees of freedom. Phys. Rev. Lett. 117(25), 251801 (2016). https://doi.org/10.1103/PhysRevLett.117.251801. arXiv: 1607.06821

26. T. Yanagida, Horizontal gauge symmetry and masses of neutrinos. Conf. Proc. C 7902131, 95-99 (1979)

27. A. Badin, A.A. Petrov, Searching for light dark matter in heavy meson decays. Phys. Rev. D 82, 034005 (2010). https://doi.org/10. 1103/PhysRevD.82.034005. arXiv: 1005.1277

28. A. Kozachuk, D. Melikhov, N. Nikitin, Rare FCNC radiative leptonic $B_{s, d} \rightarrow \gamma l^{+} l^{-}$decays in the standard model. Phys. Rev. D 97(5), 053007 (2018). https://doi.org/10.1103/PhysRevD.97. 053007. arXiv: 1712.07926

29. B. Bhattacharya, C.M. Grant, A.A. Petrov, Invisible widths of heavy mesons. Phys. Rev. D 99(9), 093010 (2019). https://doi.org/ 10.1103/PhysRevD.99.093010. arXiv:1809.04606

30. C.L. Hsu et al., Search for $B^{0}$ decays to invisible final states. Phys. Rev. D 86, 032002 (2012). https://doi.org/10.1103/PhysRevD.86. 032002. arXiv: 1206.5948

31. J.P. Lees et al., Improved Limits on $B^{0}$ Decays to Invisible Final States and to $v \bar{v} \gamma$. Phys. Rev. D 86, 051105 (2012). https://doi.org/ 10.1103/PhysRevD.86.051105. arXiv:1206.2543

32. E. Kou, et al., The Belle II Physics Book (2018). arXiv:1808.10567

33. A. Atre, T. Han, S. Pascoli, B. Zhang, The search for heavy Majorana neutrinos. JHEP 05, 030 (2009). https://doi.org/10.1088/ 1126-6708/2009/05/030. arXiv:0901.3589

34. P.B. Pal, Dirac, Majorana and Weyl fermions. Am. J. Phys. 79, 485498 (2011). https://doi.org/10.1119/1.3549729. arXiv:1006.1718

35. P.F. de Salas, D.V. Forero, C.A. Ternes, M. Tortola, J.W.F. Valle, Status of neutrino oscillations, $3 \sigma$ hint for normal mass ordering and improved CP sensitivity. Phys. Lett. B 782(2018), 633-640 (2018). https://doi.org/10.1016/j.physletb.2018.06.019. arXiv: 1708.01186

36. Belle-ECL, et al., Electromagnetic calorimeter for belle ii. J. Phys. Conf. Ser. 587(1), 012045 (2015). http://stacks.iop.org/ $1742-6596 / 587 / \mathrm{i}=1 / \mathrm{a}=012045$

37. B. Shwartz, B.I. calorimeter group, Electromagnetic calorimeter of the belle ii detector. J. Phys. Conf. Ser. 928(1), 012021 (2017). http://stacks.iop.org/1742-6596/928/i=1/a=012021 
38. M. Drewes, B. Garbrecht, Combining experimental and cosmological constraints on heavy neutrinos. Nucl. Phys. B 921, 250-315 (2017). https://doi.org/10.1016/j.nuclphysb.2017. 05.001. arXiv: 1502.00477

39. DELPHI Collaboration, Search for neutral heavy leptons produced in z decays, Zeitschrift für Physik C Particles and Fields 74(1), 5771 (1997). https://doi.org/10.1007/s002880050370

40. O. Cakir, B. Sirvanli, Rare B(s) $\longrightarrow$ nu anti-nu gamma decay beyond the standard model. Acta Phys. Polon. B 34, 2643-2650 (2003). arXiv:hep-ph/0210019

41. C.-D. Lü, D.-X. Zhang, $b_{s}\left(b_{d}\right) \rightarrow \gamma v \bar{v}$. Phys. Lett. B 381(1), 348-352 (1996). https://doi.org/10.1016/ 0370-2693(96)00587-4. http://www.sciencedirect.com/science/ article/pii/0370269396005874

42. T.M. Aliev, A. Ozpineci, M. Savci, Rare B $\rightarrow$ neutrino anti-neutrino gamma decay in light cone QCD sum rule. Phys. Lett. B 393, 143-148 (1997). https://doi.org/10.1016/ S0370-2693(96)01598-5. arXiv:hep-ph/9610255

43. E. Fernandez-Martinez, J. Hernandez-Garcia, J. Lopez-Pavon, Global constraints on heavy neutrino mixing. JHEP 08, 033 (2016). https://doi.org/10.1007/JHEP08(2016)033. arXiv:1605.08774

44. A. Abada, V. De Romeri, M. Lucente, A.M. Teixeira, T. Toma, Effective Majorana mass matrix from tau and pseudoscalar meson lepton number violating decays. JHEP 02, 169 (2018). https://doi. org/10.1007/JHEP02(2018)169. arXiv:1712.03984
45. C. Kane, C. Lehner, S. Meinel, A. Soni, Radiative leptonic decays on the lattice, in: 37th International Symposium on Lattice Field Theory, 2019. arXiv:1907.00279

46. A. Desiderio, et al., First lattice calculation of radiative leptonic decay rates of pseudoscalar mesons (6 2020). arXiv:2006.05358

47. T. Inami, C. S. Lim, Effects of Superheavy Quarks and Leptons in Low-Energy Weak Processes $\mathrm{k}(\mathrm{L}) \longrightarrow \mathrm{mu}$ anti-mu, $\mathrm{K}+\longrightarrow$ pi+ Neutrino anti-neutrino and $\mathrm{K} 0<->$ anti-K0, Prog. Theor. Phys. 65 (1981) 297, [Erratum: Prog. Theor. Phys.65,1772(1981)]. 10.1143/PTP.65.297

48. G. Buchalla, A.J. Buras, QCD corrections to rare K and B decays for arbitrary top quark mass. Nucl. Phys. B 400, 225-239 (1993). https://doi.org/10.1016/0550-3213(93)90405-E

49. A. J. Buras, Weak Hamiltonian, CP violation and rare decays, in: Probing the standard model of particle interactions. Proceedings, Summer School in Theoretical Physics, NATO Advanced Study Institute, 68th session, Les Houches, France, July 28-September 5, 1997. Pt. 1, 2, 1998, pp. 281-539. arXiv:hep-ph/9806471

50. R.J. Dowdall, C.T.H. Davies, R.R. Horgan, C.J. Monahan, J. Shigemitsu, B-meson decay constants from improved lattice nonrelativistic QCD with physical u, d, s, and c quarks. Phys. Rev. Lett. 110(22), 222003 (2013). https://doi.org/10.1103/PhysRevLett. 110.222003. arXiv:1302.2644

51. M. Tanabashi et al., Review of Particle Physics. Phys. Rev. D 98(3), 030001 (2018). https://doi.org/10.1103/PhysRevD.98.030001

52. R.A. Horn, C.R. Johnson, Matrix Analysis, 2nd edn. (Cambridge University Press, USA, 2012) 